TRANSACTIONS OF THE

AMERICAN MATHEMATICAL SOCIETY

Volume 350, Number 6, June 1998, Pages 2525-2546

S 0002-9947(98)02078-9

\title{
GEOMETRIC ASPECTS OF MULTIPARAMETER SPECTRAL THEORY
}

\author{
LUZIUS GRUNENFELDER AND TOMAŽ KOŠIR
}

\begin{abstract}
The paper contains a geometric description of the dimension of the total root subspace of a regular multiparameter system in terms of the intersection multiplicities of its determinantal hypersurfaces. The new definition of regularity used here is proved to restrict to the familiar definition in the linear case. A decomposability problem is also considered. It is shown that the joint root subspace of a multiparameter system may be decomposable even when the root subspace of each member is indecomposable.
\end{abstract}

\section{INTRODUCTION}

In this paper, we are mainly interested in a geometric description of the dimensions of the root subspaces (which are also called generalized or algebraic eigenspaces) of regular multiparameter systems of arbitrary degree. Binding and Browne studied in [3] a special class of linear two-parameter systems over the real numbers, and showed that the dimension of the root subspace at a given eigenvalue is equal to the sum of the orders of contact of the eigencurves at that eigenvalue. In this special case our results reduce to those of [3]. In [3] analytical methods were used, while we shall use results from algebraic geometry [17] and commutative algebra [1], [16].

The study of eigenspaces and root subspaces is one of the central topics of multiparameter spectral theory. Its importance stems primarily from classical analysis, where the understanding of root subspaces yields various completeness and expansion results. The literature on the subject is extensive, and for further details we refer to the books [2], [9], [14], [19], [21]. Beside this 'classical' motivation our topic may also be of algebraic and geometric interest. For example, the somewhat related problem of linearization of polynomials in several variables was studied in [5], [6], [20].

An $n$-parameter system $\mathbf{f}$ is a system of $n$ endomorphisms $f_{i}: A \otimes V_{i} \rightarrow A \otimes V_{i}$ of free $\mathcal{A}$-modules, where we assume that $A$ is a commutative regular Noetherian algebra of Krull dimension $\operatorname{dim} A=n$ over a field $F$ and $V_{i}$ are finite dimensional vector spaces over $F$. Typically, $A=F[\mathbf{x}]$ is the polynomial algebra in $n$ variables $\mathbf{x}=\left(x_{1}, x_{2}, \ldots, x_{n}\right)$ and $f_{i}$ are polynomials in $\mathbf{x}$ whose coefficients are linear maps on $V_{i}$. We say that $\mathbf{f}$ is regular if the determinants $\left\{\operatorname{det} f_{i} \mid i=1,2, \ldots, n\right\}$ form a regular sequence in $A$. Another equivalent definition is that $\mathbf{f}$ is regular if the

Received by the editors September 26, 1996.

1991 Mathematics Subject Classification. Primary 13H15, 14C17, 15A54.

Research supported in part by the NSERC of Canada and by the Ministry of Science and Technology of Slovenia. 
Koszul complex $K_{A}(\operatorname{det} \mathbf{f})$ is acyclic [17]. We show that in the particular case when $A=F[\mathbf{x}]$ and all the $f_{i}$ are linear polynomials this definition coincides with the usual definition of regularity that is used, for instance, by Atkinson in [2]. Our definition of regularity not only extends the familiar one to the nonlinear case, but is also natural in the context of the commutative algebra used in our proofs. Another advantage is that it can be localized, and so we can speak of local regularity at each eigenvalue. Since our results are local in nature, it suffices to assume local regularity of the systems. One of the crucial results is that regularity of the system $\mathbf{f}$ at a point $\mathfrak{m}$ in its spectrum $\sigma(\mathbf{f})$ implies acyclicity of its Koszul complex $K_{\mathcal{A}}(\mathbf{f})$, where $\mathcal{A}$ is the localization of $A$ at $\mathfrak{m}$. In [10] we proved that the root subspace of a linear multiparameter system at a point $\mathfrak{m} \in \sigma(\mathbf{f})$ is given by the cotensor product

$$
\mathcal{N}=\mathcal{M}_{1}^{0} \otimes \mathcal{A}^{0} \mathcal{M}_{2}^{0} \otimes \mathcal{A}^{0} \ldots \otimes^{\mathcal{A}^{0}} \mathcal{M}_{n}^{0},
$$

where $\mathcal{M}_{i}$ is the cokernel of $f_{i}$ localized at $\mathfrak{m}$ and where ${ }^{0}$ is the contravariant functor which takes a module into its dual comodule [12]. The left adjoint of ${ }^{0}$ is * and the composite ${ }^{0 *}$ is the completion functor for the cofinite topology. Hence, for every $\mathcal{A}^{0}$-comodule $\mathcal{N}$ the dual $\mathcal{M}=\mathcal{N}^{*}$ is a module over a complete local ring, so that we can apply Serre's multiplicity theory [17] to compute the length $l^{\mathcal{A}}$ of $\mathcal{M}$. Our main result is that

$$
l^{\mathcal{A}}(\mathcal{M})=l^{\mathcal{A}}\left(\mathcal{A} /\left\langle\operatorname{det} f_{1}, \ldots, \operatorname{det} f_{n}\right\rangle\right),
$$

which in turn is the intersection multiplicity of the system of determinantal hypersurfaces at the point $\mathfrak{m}$. In the case $A=F[\mathbf{x}]$ and $\mathfrak{m}=\left\langle x_{1}-\lambda_{1}, x_{2}-\lambda_{2}, \ldots, x_{n}-\lambda_{n}\right\rangle$ the residue class field of $\mathcal{A}$ is the fixed underlying field $F$; therefore the length coincides with the dimension of $\mathcal{M}$ as a vector space over $F$. For a general maximal ideal $\mathfrak{m}$ of $\mathcal{A}$ we have $\operatorname{dim}_{F} \mathcal{M}=l^{\mathcal{A}}(\mathcal{M})[\mathcal{A} / \mathfrak{m}: F]$. In this paper we mostly consider the case when $\mathcal{A} / \mathfrak{m} \cong F$.

For the terminology from commutative algebra used in this paper we refer to the books of Matsumura [16] and Serre [17], while for the terminology concerning the coalgebra dual of the polynomial algebra we refer to our papers [10], [11]. All algebras and coalgebras are over a fixed base field $F$. If $A$ is a commutative $F$ algebra and $M$ is an $A$-module, then $\operatorname{Max}(A)$ and $\operatorname{Spec}(A)$ are the maximal ideal spectrum and the prime ideal spectrum of $A$, while $\operatorname{Var}(M)=\left\{\mathfrak{p} \in \operatorname{Spec}(A) \mid M_{\mathfrak{p}} \neq\right.$ $0\}=\{\mathfrak{p} \in \operatorname{Spec}(A) \mid \operatorname{ann} M \subseteq \mathfrak{p}\}$ is the variety of $M$.

\section{Spectral Properties of an Endomorphism}

For any regular commutative Noetherian algebra $A$ over the field $F$ and any finite dimensional vector space $V$ there is a canonical isomorphism of $A$-algebras

$$
A \otimes \operatorname{End}_{F}(V) \cong \operatorname{End}_{A}(A \otimes V) .
$$

For every element $f \in A \otimes \operatorname{End}_{F}(V)$, consider the exact sequence

$$
A \otimes V \stackrel{f}{\rightarrow} A \otimes V \rightarrow M \rightarrow 0
$$

and its localization at a maximal ideal $\mathfrak{m}$ of $A$

$$
\mathcal{A} \otimes V \stackrel{f}{\rightarrow} \mathcal{A} \otimes V \rightarrow \mathcal{M} \rightarrow 0,
$$

where the Noetherian local algebra $\mathcal{A}=A_{\mathfrak{m}}$ has maximal ideal $\mathfrak{m} \mathcal{A}$ and the residue class field $k=\mathcal{A} / \mathfrak{m} \mathcal{A}$ is a finite field extension of $F$. The coalgebra dual $\mathcal{A}^{0}$ is a 
colocal coalgebra and $\widehat{\mathcal{A}}=\mathcal{A}^{0 *}$ is the completion of $\mathcal{A}$ in its $\mathfrak{m} \mathcal{A}$-adic topology. The comodule dual sequence

$$
0 \rightarrow \mathcal{M}^{0} \rightarrow \mathcal{A}^{0} \otimes V \stackrel{f^{0}}{\longrightarrow} \mathcal{A}^{0} \otimes V
$$

is exact, since the functor ${ }^{0}: \operatorname{Mod}_{\mathcal{A}}^{o p} \rightarrow \operatorname{Comod}_{\mathcal{A}^{0}}$ is a right adjoint [12]. Our main interest lies with $\mathcal{M}^{0}$. But $\mathcal{M}^{0 * 0}=\mathcal{M}^{0}$ and the completion functor ${ }^{0 *}=\widehat{\mathcal{A}} \otimes_{\mathcal{A}}-$ : $\operatorname{Mod}_{\mathcal{A}} \rightarrow \operatorname{Mod}_{\widehat{\mathcal{A}}}$ is exact, so that we may always replace $\mathcal{A}$ by its completion $\mathcal{A}^{0 *}$. Since $A$ is regular the localization $\mathcal{A}=A_{\mathfrak{m}}$ is also regular [17, p. IV-41, Prop. 23], and $\mathcal{A}$ is a unique factorization domain [17, IV-39].

The spectrum of the $A$-endomorphism $f: A \otimes V \rightarrow A \otimes V$ is defined by

$$
\sigma(f)=\operatorname{Max}(A) \cap \operatorname{Var}(M) .
$$

In this paper we study spectral properties of the root subspaces of one and several endomorphisms, and we will therefore mainly consider the localized exact sequences above.

For an element $p \in A$ we denote by $\langle p\rangle$ the ideal generated by $p$ in the localized algebra $\mathcal{A}=A_{\mathfrak{m}}$. If $V$ is finite dimensional and $\operatorname{det} f$ is a regular element of $\mathcal{A}$, then $f(\operatorname{adj} f)=(\operatorname{det} f) \otimes 1$. The commutative diagram with exact rows of $\mathcal{A}$-modules

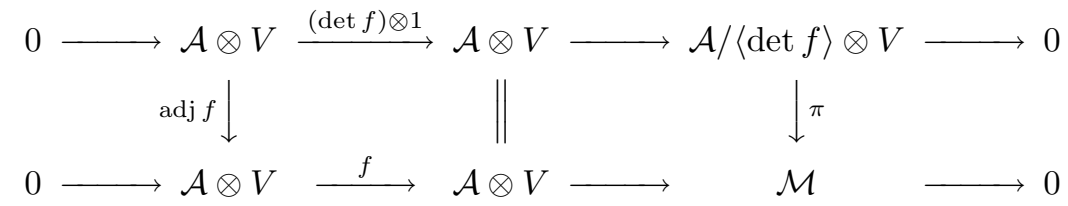

induces a commutative diagram with exact rows of $\mathcal{A}^{0}$-comodules

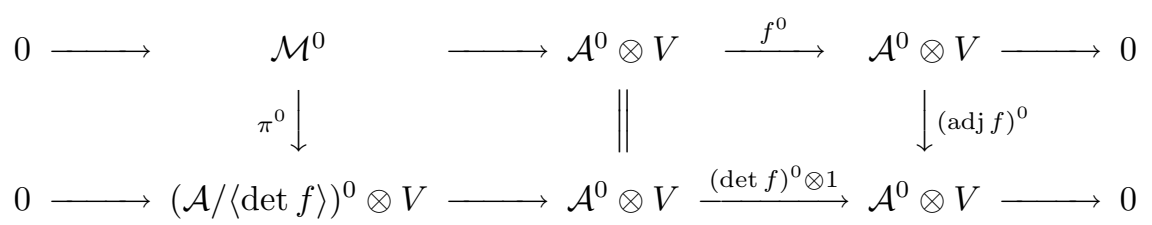

and therefore $(\mathcal{A} /\langle\operatorname{det} f\rangle)^{0} \cong \operatorname{ker}\left((\operatorname{det} f)^{0}\right)$. Observe that the $\mathcal{A}$-module map $\pi$ : $\mathcal{A} /\langle\operatorname{det} f\rangle \otimes V \rightarrow \mathcal{M}$ is surjective.

2.1. Theorem. Suppose that $\mathcal{A}$ is regular, hence a unique factorization domain, and that $\operatorname{det} f=p_{1}^{l_{1}} p_{2}^{l_{2}} \ldots p_{r}^{l_{r}}$ is the primary decomposition in $\mathcal{A}$ of the determinant of $f \in \mathcal{A} \otimes \operatorname{End}_{F}(V)$. If $\mathfrak{p}_{i}=\left\langle p_{i}\right\rangle$ for $i=1,2, \ldots, r$, then $\left\{\mathfrak{p}_{1}, \mathfrak{p}_{2}, \ldots, \mathfrak{p}_{n}\right\}$ is the set of minimal primes associated with $\mathcal{M},\left\{\mathfrak{p}_{1}, \mathfrak{p}_{2}, \ldots, \mathfrak{p}_{r}\right\} \subset \operatorname{Ass} \mathcal{M} \subset \operatorname{Var} \mathcal{M}$, and $l_{i}=l_{\mathfrak{p}_{i}}(\mathcal{M})$ is the length of the $\mathcal{A}_{\mathfrak{p}_{i}}$-module $\mathcal{M}_{\mathfrak{p}_{i}}$. Moreover, if $\operatorname{dim} \mathcal{A}=2$, then $\operatorname{Ass} \mathcal{M}=\operatorname{Var} \mathcal{M} \backslash\{\mathfrak{m}\}$.

Proof. For every prime ideal $\mathfrak{p}$ of $\mathcal{A}$ the sequence of $\mathcal{A}_{\mathfrak{p}}$-modules

$$
\mathcal{A}_{\mathfrak{p}} \otimes V \stackrel{f_{\mathfrak{p}}}{\longrightarrow} \mathcal{A}_{\mathfrak{p}} \otimes V \rightarrow \mathcal{M}_{\mathfrak{p}} \rightarrow 0
$$

is exact and $f(\operatorname{adj} f)=(\operatorname{det} f) \otimes 1$ has localization $f_{\mathfrak{p}}\left(\operatorname{adj} f_{\mathfrak{p}}\right)=\left(\operatorname{det} f_{\mathfrak{p}}\right) \otimes 1$. Observe that $\operatorname{det} f_{\mathfrak{p}}$ is invertible in $\mathcal{A}_{\mathfrak{p}}$ if and only if $p_{i} \notin \mathfrak{p}$ for $i=1,2, \ldots, r$, i.e. $\operatorname{det} f_{\mathfrak{p}}$ is not invertible in $\mathcal{A}_{\mathfrak{p}}$ if and only if $p_{i} \in \mathfrak{p}$ for some $i$. Hence, $\mathcal{M}_{\mathfrak{p}} \neq 0$ if and only if $p_{i} \in \mathfrak{p}$ for some $i$. Moreover, $\mathcal{M}_{\mathfrak{p}_{i}} \neq 0$ for all $i$, so that $\mathfrak{p}_{i} \in \operatorname{Var} \mathcal{M}$ for every $i$. This implies that $\mathfrak{p} \in \operatorname{Var} \mathcal{M}$ if and only if $p_{i} \in \mathfrak{p}$ and hence $\left\{\mathfrak{p}_{1}, \mathfrak{p}_{2}, \ldots, \mathfrak{p}_{r}\right\} \subset \operatorname{Ass} \mathcal{M}$. If $\mathfrak{p} \in \operatorname{Var} \mathcal{M}$ and $\operatorname{dim} \mathcal{A} / \mathfrak{p}=n-1$, then $\mathfrak{p}=\mathfrak{p}_{i}$ for some $i$. If $\operatorname{dim} \mathcal{A}=2$, then 
Ass $\mathcal{M}=\operatorname{Var} \mathcal{M} \backslash\{\mathfrak{m}\}$. This is because $\mathcal{M}_{\mathfrak{p}} \neq 0$ if and only $p_{i} \in \mathfrak{p}$ for some $i$, therefore $\mathfrak{p}_{i} \subset \mathfrak{p} \subset \mathfrak{m}$, which implies that $\mathfrak{p}_{i}=\mathfrak{p}$ or $\mathfrak{p}=\mathfrak{m}$.

Now observe that $\operatorname{dim} \mathcal{A}_{\mathfrak{p}_{i}}=1$, since every nonzero principal prime ideal of a unique factorization domain has height one. Thus, $\mathcal{A}_{\mathfrak{p}_{i}}$ is a discrete valuation ring, i.e. a local Noetherian domain whose maximal ideal is principal, while

$$
\operatorname{dim} \mathcal{M}=\operatorname{dim} \mathcal{A} / \operatorname{ann} \mathcal{M}=\operatorname{dim} \mathcal{A} / \mathfrak{p}_{i}=\operatorname{coht} \mathfrak{p}_{i}=\operatorname{dim} \mathcal{A}-1 .
$$

In the exact sequence of $\mathcal{A}_{\mathfrak{p}_{i}}$-modules

$$
\mathcal{A}_{\mathfrak{p}_{i}} \otimes V \stackrel{f_{\mathfrak{p}_{i}}}{\longrightarrow} \mathcal{A}_{\mathfrak{p}_{i}} \otimes V \rightarrow \mathcal{M}_{\mathfrak{p}_{i}} \rightarrow 0
$$

the module $\mathcal{M}_{\mathfrak{p}_{i}}$ is not zero and $\operatorname{det} f_{\mathfrak{p}_{i}}=c_{i} p_{i}^{l_{i}}$ for some invertible element $c_{i}$ in $\mathcal{A}_{\mathfrak{p}_{i}}$. Since $\mathcal{A}_{\mathfrak{p}_{i}}$ is a discrete valuation ring, in particular a principal ideal domain, the map $f_{\mathfrak{p}_{i}}$ can be diagonalized, i.e. $\mathcal{M}_{\mathfrak{p}_{i}} \cong \bigoplus_{j} \mathcal{A}_{\mathfrak{p}_{i}} / p_{i}^{d_{i j}} \mathcal{A}$ for some integers $d_{i 0} \geq d_{i 1} \geq \ldots \geq d_{i s}$, and $l_{\mathfrak{p}_{i}}(\mathcal{M})=l\left(\mathcal{M}_{\mathfrak{p}_{i}}\right)=\sum_{j=1}^{s} d_{i j}=l_{i}$.

Let us mention at this point that for every $\mathcal{A}$-module map $f: \mathcal{A} \otimes V \rightarrow \mathcal{A} \otimes V$ the equality $f(\operatorname{adj} f)=(\operatorname{det} f) \otimes 1$ implies that $\langle\operatorname{det} f\rangle \subseteq \operatorname{ann} \mathcal{M} \subset \bigcap_{i} \mathfrak{p}_{i}=\left\langle p_{1} p_{2} \cdots p_{r}\right\rangle$, where $\operatorname{det} f=p_{1}^{l_{1}} p_{2}^{l_{2}} \ldots p_{r}^{l_{r}}$ is the primary decomposition in $\mathcal{A}$ and $\mathfrak{p}_{i}=\left\langle p_{i}\right\rangle$.

2.2. Theorem. If $\mathfrak{m} \in \sigma(f)$ is simple, i.e. if $\mathcal{M} / \mathfrak{m} \mathcal{M} \cong \mathcal{A} / \mathfrak{m} \mathcal{A}$, then $\mathcal{M} \cong$ $\mathcal{A} /\langle\operatorname{det} f\rangle$.

Proof. The maximal ideal of $\mathcal{A}=A_{\mathfrak{m}}$ is $\mathfrak{m} \mathcal{A}$, and $\mathcal{A} / \mathfrak{m} \mathcal{A} \otimes_{\mathcal{A}} \mathcal{M} \cong \mathcal{M} / \mathfrak{m} \mathcal{M}$. Every homomorphism $\theta: \mathcal{A} / \mathfrak{m} \mathcal{A} \rightarrow \mathcal{M} / \mathfrak{m} \mathcal{M}$ can be lifted to an $\mathcal{A}$-module map $\chi: \mathcal{A} \rightarrow$ $\mathcal{M}$ such that the diagram

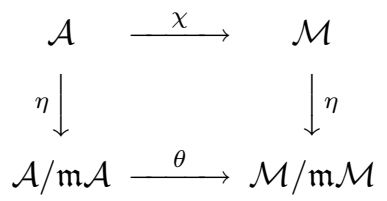

commutes, and so $1 \otimes_{\mathcal{A}} \chi=\theta$ (via the natural isomorphism $\mathcal{A} / \mathfrak{m} \mathcal{A} \otimes_{\mathcal{A}} \mathcal{A} \cong \mathcal{A} / \mathfrak{m} \mathcal{A}$ ). Tensoring the exact sequence of $\mathcal{A}$-modules

$$
\mathcal{A} \stackrel{\chi}{\rightarrow} \mathcal{M} \rightarrow \operatorname{cok} \chi \rightarrow 0
$$

by $\mathcal{A} / \mathfrak{m} \mathcal{A}$ over $\mathcal{A}$ gives a commutative diagram

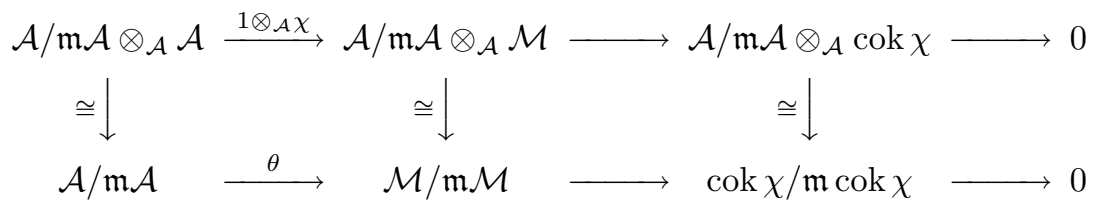

with exact rows. If $\theta: \mathcal{A} / \mathfrak{m} \mathcal{A} \rightarrow \mathcal{M} / \mathfrak{m} \mathcal{M}$ is an isomorphism, then $\operatorname{cok} \chi / \mathfrak{m} \operatorname{cok} \chi=$ 0 . By the Nakayama Lemma [1] we must conclude that $\operatorname{cok} \chi=0$, so that $\chi$ : $\mathcal{A} \rightarrow \mathcal{M}$ is surjective and $\operatorname{ker} \chi=\operatorname{ann} \mathcal{M}$. If $\operatorname{det} f=p_{1}^{l_{1}} p_{2}^{l_{2}} \cdots p_{r}^{l_{r}}$ is the primary decomposition then it follows from Theorem 2.1 that $\mathfrak{p}_{i}=\left\langle p_{i}\right\rangle \in \operatorname{Ass} \mathcal{M}$. Then $\mathcal{A}_{\mathfrak{p}_{i}}$ is a discrete valuation ring for each $i$, and hence $(\operatorname{ann} \mathcal{M})_{\mathfrak{p}_{i}}=\operatorname{ann}\left(\mathcal{M}_{\mathfrak{p}_{i}}\right)=p_{i}^{s_{i}} \mathcal{A}_{\mathfrak{p}_{i}}$ for some $s_{i} \leq l_{i}$. On the other hand, because $\mathcal{M}_{\mathfrak{p}_{i}}$ can be generated as an $\mathcal{A}_{\mathfrak{p}_{i}}$ module by a single element, it follows that the map $f_{\mathfrak{p}_{i}}$ in the exact sequence

$$
\mathcal{A}_{\mathfrak{p}_{i}} \otimes V \stackrel{f_{\mathfrak{p}_{i}}}{\longrightarrow} \mathcal{A}_{\mathfrak{p}_{i}} \otimes V \rightarrow \mathcal{M}_{\mathfrak{p}_{i}} \rightarrow 0
$$


has a diagonal matrix representation with diagonal $\left(p_{i}^{s_{i}}, 1, \ldots, 1\right)$. But then $s_{i}=l_{i}$, since $\operatorname{det}\left(f_{p_{i}}\right)=c_{i} p_{i}^{l_{i}}$ for some invertible element $c_{i}$ in $\mathcal{A}_{\mathfrak{p}_{i}}$, and so ann $\mathcal{M}_{\mathfrak{p}_{i}}=$ $p_{i}^{l_{i}} \mathcal{A}_{\mathfrak{p}_{i}}$. Moreover, if $a \in \operatorname{ann} \mathcal{M}$, i.e. $a \mathcal{M}=0$, then $a \mathcal{M}_{\mathfrak{p}_{i}}=0$; hence $a \in$ ann $\mathcal{M}_{\mathfrak{p}_{i}}=p_{i}^{l_{i}} \mathcal{A}_{\mathfrak{p}_{i}}$ for every $i$, which implies that each $p_{i}^{l_{i}}$ divides $a$ in $\mathcal{A}$, so that $a \in\langle\operatorname{det} f\rangle$. We conclude that $\operatorname{ker} \chi=\operatorname{ann} \mathcal{M}=\langle\operatorname{det} f\rangle$ and $\mathcal{A} /\langle\operatorname{det} f\rangle \cong \mathcal{M}$.

The above theorem remains valid when $\mathcal{A}$ and $\mathcal{M}$ are replaced by $\mathcal{A} / \mathfrak{m}^{i} \mathcal{A}$ and $\mathcal{M} / \mathfrak{m}^{i} \mathcal{M}$ for $i \geq 1$. It follows that the isomorphism $\mathcal{A} /\langle\operatorname{det} f\rangle \cong \mathcal{M}$ is an isomorphism of filtered $\mathcal{A}$-modules.

\section{Finite Systems of Endomorphisms}

Consider a system $\mathbf{f}=\left(f_{1}, f_{2}, \ldots, f_{n}\right)$ of endomorphisms $f_{i} \in \operatorname{End}_{A}\left(A \otimes V_{i}\right)$, where the $V_{i}$ are finite dimensional vector spaces over the field $F$. Note that $n=$ $\operatorname{dim} A$. For each $f_{i}$ consider the exact sequence

$$
A \otimes V_{i} \stackrel{f_{i}}{\longrightarrow} A \otimes V_{i} \rightarrow M_{i} \rightarrow 0
$$

and its localization

$$
\mathcal{A} \otimes V_{i} \stackrel{f_{i}}{\longrightarrow} \mathcal{A} \otimes V_{i} \rightarrow \mathcal{M}_{i} \rightarrow 0
$$

at a maximal ideal $\mathfrak{m}$ of $A$. Then the comodule dual sequence

$$
0 \rightarrow \mathcal{M}_{i}^{0} \rightarrow \mathcal{A}^{0} \otimes V_{i} \stackrel{f_{i}^{0}}{\longrightarrow} \mathcal{A}^{0} \otimes V_{i}
$$

is also exact. The joint spectrum of $\mathbf{f}$ is the intersection

$$
\sigma(\mathbf{f})=\bigcap_{i=1}^{n} \sigma\left(f_{i}\right)=\operatorname{Max}(A) \bigcap_{i=1}^{n} \operatorname{Var}\left(M_{i}\right)
$$

and consists of all maximal ideals $\mathfrak{m}$ of $A$ satisfying $\left(M_{i}\right)_{\mathfrak{m}} \neq 0$ for $i=1,2, \ldots, n$. If $A=F[\mathbf{x}]$ and $\mathfrak{m}=\left\langle x_{1}-\lambda_{1}, x_{2}-\lambda_{2}, \ldots, x_{n}-\lambda_{n}\right\rangle$, then $\boldsymbol{\lambda}=\left(\lambda_{1}, \lambda_{2}, \ldots, \lambda_{n}\right)$ is an eigenvalue of $\mathbf{f}$ in the sense usual in multiparameter spectral theory [2], [3], [10], [11], [14]. In this case we will frequently change the notation and write $\boldsymbol{\lambda}$ for the ideal $\mathfrak{m}$.

The Koszul complex $K_{A}(\mathbf{f})$ associated with $\mathbf{f}$ is defined recursively by

$$
K_{A}\left(f_{i}\right): A \otimes V_{i} \stackrel{f_{i}}{\longrightarrow} A \otimes V_{i}
$$

and

$$
K_{A}\left(f_{1}, \ldots, f_{i}, f_{i+1}\right)=K_{A}\left(f_{1}, \ldots, f_{i}\right) \otimes_{A} K_{A}\left(f_{i+1}\right) .
$$

Its homology is denoted by $H_{*}(\mathbf{f})$. In a similar way we construct the Koszul complex $K_{A}(\operatorname{det} \mathbf{f})$ of the $n$-tuple $\operatorname{det} \mathbf{f}=\left(\operatorname{det} f_{1}, \operatorname{det} f_{2}, \ldots, \operatorname{det} f_{n}\right)$ of elements of $A$ recursively by

$$
K_{A}\left(\operatorname{det} f_{i}\right): A \stackrel{\operatorname{det} f_{i}}{\longrightarrow} A
$$

and

$$
K_{A}\left(\operatorname{det} f_{1}, \ldots, \operatorname{det} f_{i}, \operatorname{det} f_{i+1}\right)=K_{A}\left(\operatorname{det} f_{1}, \operatorname{det} f_{2}, \ldots, \operatorname{det} f_{i}\right) \otimes_{A} K_{A}\left(\operatorname{det} f_{i+1}\right),
$$

and its homology is $H_{*}(\operatorname{det} \mathbf{f})$. Since localization and completion are exact and preserve tensor products, we see that $K_{A}(\mathbf{f})_{\mathfrak{m}} \cong K_{\mathcal{A}}(\mathbf{f})$ and $K_{A}(\operatorname{det} \mathbf{f})_{\mathfrak{m}} \cong K_{\mathcal{A}}(\operatorname{det} \mathbf{f})$ 
at the maximal ideal $\mathfrak{m}$ of $A$. Moreover, $H_{*}\left(K_{\mathcal{A}}(\mathbf{f})\right) \cong H_{*}\left(K_{A}(\mathbf{f})\right)_{\mathfrak{m}}$ and, in particular,

$$
H_{0}\left(K_{\mathcal{A}}(\mathbf{f})\right) \cong H_{0}\left(K_{A}(\mathbf{f})_{\mathfrak{m}}\right) \cong \mathcal{M}_{1} \otimes_{\mathcal{A}} \mathcal{M}_{2} \otimes_{\mathcal{A}} \ldots \otimes_{\mathcal{A}} \mathcal{M}_{n}
$$

A chain complex $K$ is called acyclic if $H_{p}(K)=0$ for all $p \neq 0$. The system f is called regular if the Koszul complex $K_{A}(\operatorname{det} \mathbf{f})$ is acyclic. Locally, we say that $\mathbf{f}$ is regular at a maximal ideal $\mathfrak{m} \in \sigma(\mathbf{f})$ if $K_{\mathcal{A}}(\operatorname{det} \mathbf{f})$ is acyclic. By [17, IV-5-12] this is equivalent to saying that $\mathbf{f}$ is regular (locally regular, respectively) if ( $\operatorname{det} f_{1}, \operatorname{det} f_{2}, \ldots, \operatorname{det} f_{n}$ ) is a regular sequence in $A$ (in $\mathcal{A}$, respectively). An element $\mathfrak{m} \in \sigma(\mathbf{f})$ is called simple if $\mathcal{M}_{i} / \mathfrak{m} \mathcal{M}_{i} \cong \mathcal{A} / \mathfrak{m} \mathcal{A}$ for $i=1,2, \ldots, n$. The following two results are immediate consequences of Theorem 2.2 .

3.1. Corollary. A point $\mathfrak{m} \in \sigma(\mathbf{f})$ is simple if and only if

$$
\mathcal{M} \cong \mathcal{M}_{1} \otimes_{\mathcal{A}} \mathcal{M}_{2} \otimes_{\mathcal{A}} \ldots \otimes_{\mathcal{A}} \mathcal{M}_{n} \cong \mathcal{A} /\left\langle\operatorname{det} f_{1}, \operatorname{det} f_{2}, \ldots, \operatorname{det} f_{n}\right\rangle
$$

as filtered $\mathcal{A}$-modules.

3.2. Corollary. If $\mathfrak{m} \in \sigma(\mathbf{f})$ is simple, then $\mathcal{M}$ is (up to isomorphisms of filtered $\mathcal{A}$-modules) uniquely determined by the determinants $\operatorname{det} f_{1}, \operatorname{det} f_{2}, \ldots, \operatorname{det} f_{n}$ in $\mathcal{A}$. In particular, the length of the $\mathcal{A}$-module $\mathcal{M} / \mathfrak{m}^{k} \mathcal{M}$ depends on the determinants only.

The Koszul complex $K_{\mathcal{A}^{0}}\left(\mathbf{f}^{0}\right)$ associated with the sequence of $\mathcal{A}^{0}$-comodule maps $\mathbf{f}^{0}$ is the cochain complex defined recursively by

$$
K_{\mathcal{A}^{0}}\left(f_{i}^{0}\right): \mathcal{A}^{0} \otimes V_{i} \rightarrow \mathcal{A}^{0} \otimes V_{i}
$$

and

$$
K_{\mathcal{A}^{0}}\left(f_{1}^{0}, \ldots, f_{i}^{0}, f_{i+1}^{0}\right)=K_{\mathcal{A}^{0}}\left(f_{1}^{0}, \ldots, f_{i}^{0}\right) \otimes \mathcal{A}^{0} K_{\mathcal{A}^{0}}\left(f_{i+1}^{0}\right) .
$$

Since the functor ${ }^{0}: \operatorname{Mod}_{\mathcal{A}} \rightarrow \operatorname{Comod}_{\mathcal{A}^{0}}$ is exact [12] and preserves tensors, it follows that $K_{\mathcal{A}^{0}}\left(\mathbf{f}^{0}\right) \cong K_{\mathcal{A}}(\mathbf{f})^{0}$ and $H^{*}\left(K_{\mathcal{A}^{0}}\left(\mathbf{f}^{0}\right)\right) \cong H_{*}\left(K_{\mathcal{A}}(\mathbf{f})\right)^{0}$. In particular,

$$
H^{0}\left(K_{\mathcal{A}^{0}}\left(\mathbf{f}^{0}\right)\right) \cong H_{0}\left(K_{\mathcal{A}}(\mathbf{f})\right)^{0} \cong \mathcal{M}_{1}^{0} \otimes \mathcal{A}^{0} \mathcal{M}_{2}^{0} \otimes^{\mathcal{A}^{0}} \ldots \otimes^{\mathcal{A}^{0}} \mathcal{M}_{n}^{0}
$$

is the 'total root space' of the system $\mathbf{f}$ at $\mathfrak{m}$.

Our main tool relating the geometric aspects of the determinants $\operatorname{det} \mathbf{f}$ to the module theoretic aspects of the $\mathcal{M}_{i}$ is Serre's multiplicity theory [17]. In addition, the following theorem is a crucial step toward our main result.

3.3. Theorem. If the system $\mathbf{f}$ is regular at a point $\mathfrak{m} \in \sigma(\mathbf{f})$, then:

1. the Koszul complex $K_{\mathcal{A}}\left(f_{1}, f_{2}, \ldots, f_{i}\right)$ is acyclic and $\mathcal{A}$-free for each $i=$ $1,2, \ldots, n$,

2. $\operatorname{Tor}_{j}^{\mathcal{A}}\left(\mathcal{M}_{1} \otimes_{\mathcal{A}} \cdots \otimes_{\mathcal{A}} \mathcal{M}_{i-1}, \mathcal{M}_{i}\right)=0$ for $i=2,3, \ldots, n$ and $j \neq 0$.

Proof. By definition, $\mathbf{f}$ is regular at the point $\mathfrak{m}$ if $K_{\mathcal{A}}(\operatorname{det} \mathbf{f})$ is acyclic. By [17, IV$5-12]$ this is equivalent to saying that $\operatorname{det} \mathbf{f}=\left(\operatorname{det} f_{1}, \operatorname{det} f_{2}, \ldots, \operatorname{det} f_{n}\right)$ is a regular sequence in $\mathcal{A}$. Since $\operatorname{det} f_{i} \neq 0$ and $f_{i}\left(\operatorname{adj} f_{i}\right)=\left(\operatorname{det} f_{i}\right) \otimes 1=\left(\operatorname{adj} f_{i}\right) f_{i}$ we have 
the commutative diagram with exact rows and columns of $\mathcal{A}$-modules

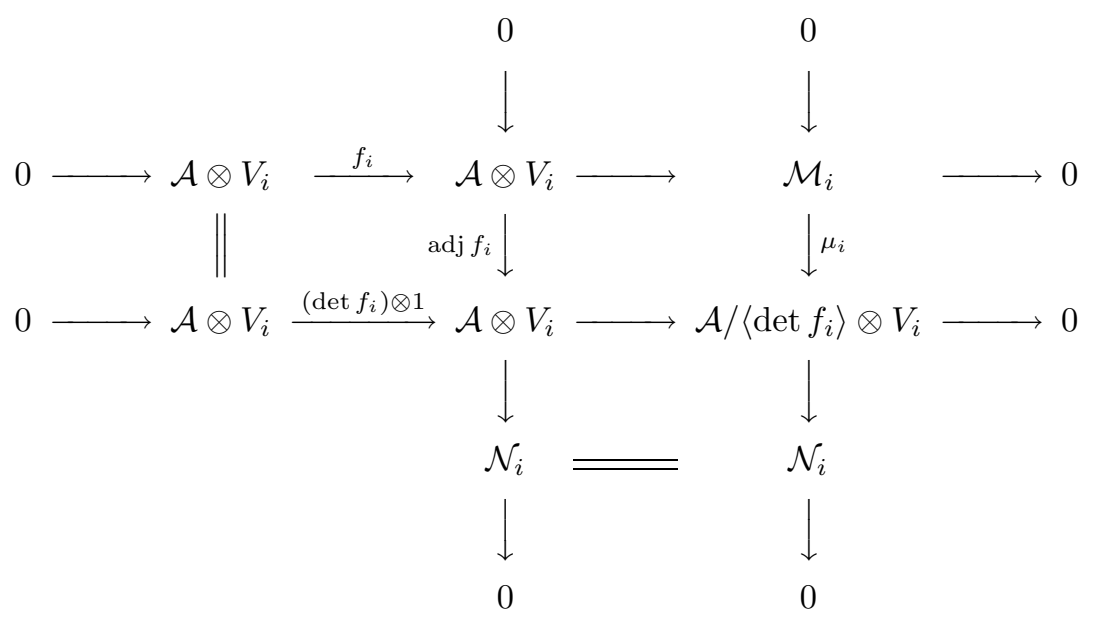

for each $i=1,2, \ldots, n$. This means in particular that

$$
0 \rightarrow \mathcal{A} \otimes V_{i} \stackrel{f_{i}}{\longrightarrow} \mathcal{A} \otimes V_{i} \rightarrow \mathcal{M}_{i} \rightarrow 0
$$

is a free $\mathcal{A}$-resolution of $\mathcal{M}_{i}$, so that

$$
\operatorname{dh}_{\mathcal{A}} \mathcal{M}_{i}=1 .
$$

But $\mathcal{A}$ is a regular Noetherian local algebra, so we also have [17, IV-35-43]

$$
\operatorname{gldh} \mathcal{A}=\operatorname{dim} \mathcal{A}=n \quad \text { and } \quad \operatorname{dh}_{\mathcal{A}} \mathcal{M}_{i}+\operatorname{codh}_{\mathcal{A}} \mathcal{M}_{i}=n .
$$

We conclude that $\operatorname{dim} \mathcal{M}_{i}=n-1=\operatorname{codh}_{\mathcal{A}} \mathcal{M}_{i}$ and similarly $\operatorname{dim} \mathcal{N}_{i}=n-1=$ $\operatorname{codh}_{\mathcal{A}} \mathcal{N}_{i}$, so that each $\mathcal{M}_{i}$ and each $N_{i}$ is a Cohen-Macaulay $\mathcal{A}$-module [17, IV-18]. Moreover, we also have the commutative diagram with exact rows and columns of $\mathcal{A}$-modules

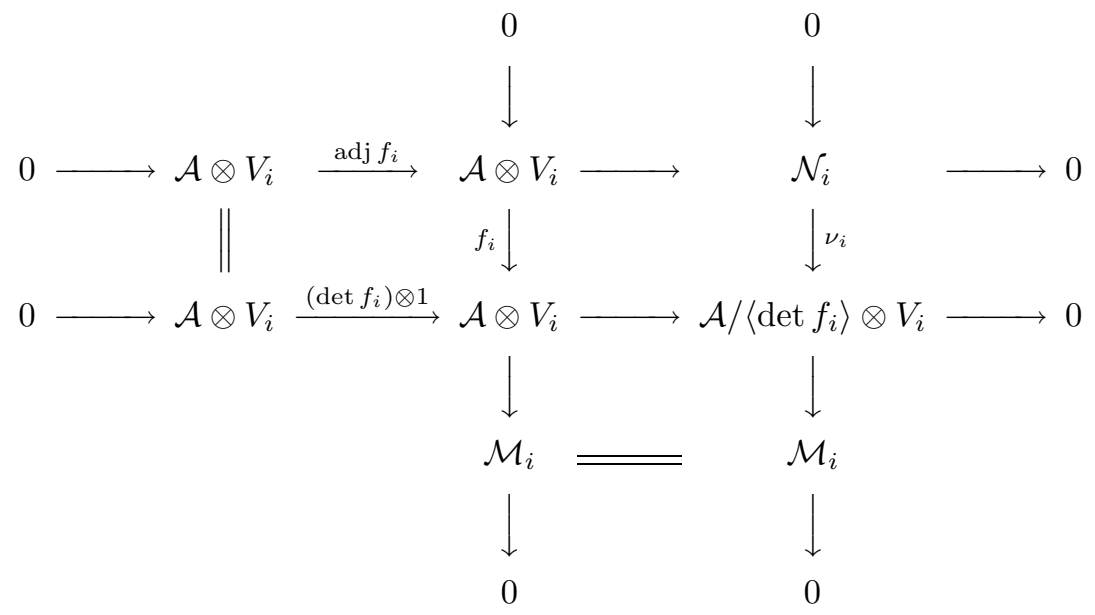

for each $i=1,2, \ldots, n$.

We want to show by induction on $i$ that the Koszul complex $K_{\mathcal{A}}\left(f_{1}, \ldots, f_{i}\right)$ for $i=1, \ldots, n$, is in fact a free resolution of $\mathcal{M}^{[i]}=\mathcal{M}_{1} \otimes_{\mathcal{A}} \cdots \otimes_{\mathcal{A}} \mathcal{M}_{i}$, i.e. that $K_{\mathcal{A}}\left(f_{1}, \ldots, f_{i}\right)$ is acyclic. So suppose that $\mathbf{X}_{i}=K_{\mathcal{A}}\left(f_{1}, \ldots, f_{i}\right)$ is acyclic and 
let $\mathbf{Y}_{i}=K_{\mathcal{A}}\left(\operatorname{det} f_{1}, \operatorname{det} f_{2}, \ldots, \operatorname{det} f_{i}\right)$, which is acyclic by hypothesis. Then by induction the map of complexes

$$
\mathbf{F}_{i}: \mathbf{X}_{i} \rightarrow \mathbf{Y}_{i}
$$

is defined as the tensor product of the injective maps $F_{j}: K_{\mathcal{A}}\left(f_{j}\right) \rightarrow K_{\mathcal{A}}\left(\operatorname{det} f_{j}\right)$ given by the commutative diagrams

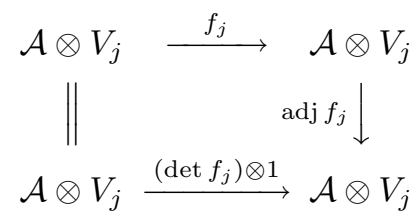

so that

$$
\mathbf{F}_{i+1}=\mathbf{F}_{i} \otimes_{\mathcal{A}} F_{i+1}: \mathbf{X}_{i+1}=\mathbf{X}_{i} \otimes_{\mathcal{A}} K_{\mathcal{A}}\left(f_{i+1}\right) \rightarrow \mathbf{Y}_{i} \otimes_{\mathcal{A}} K_{\mathcal{A}}\left(\operatorname{det} f_{i+1}\right)=\mathbf{Y}_{i+1} .
$$

This map of complexes induces a map of exact sequences in homology

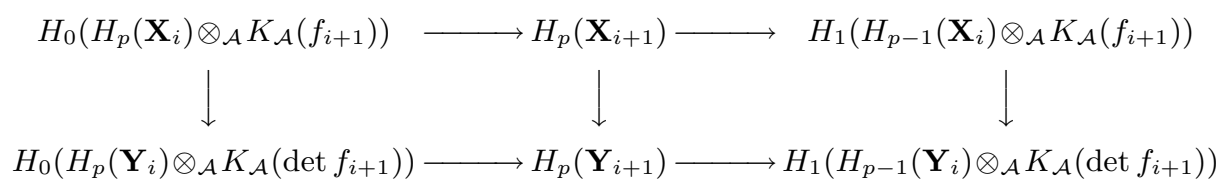

for $p \geq 0$ [17, p. IV-2, Prop. 1], where the left hand and the right hand horizontal arrows are injective and surjective, respectively. When $\mathbf{X}_{i}$ is acyclic, and since $\mathbf{Y}_{i}$ is acyclic by hypothesis, the diagram is trivial for $p>1$, so that

$$
H_{p}\left(\mathbf{X}_{i+1}\right)=0
$$

for $p>1$. The case $p=0$ reduces to the commutative square

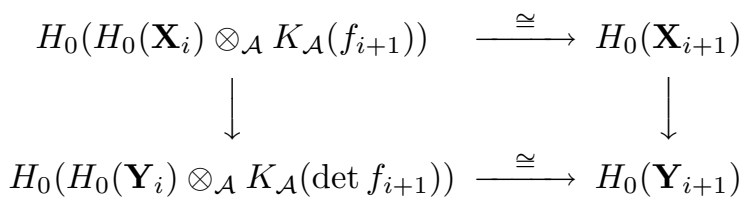

and it gives a canonical map

$$
H_{0}\left(\mathbf{F}_{i+1}\right)=\mu^{[i+1]}: \mathcal{M}^{[i+1]}=H_{0}\left(\mathbf{X}_{i+1}\right) \rightarrow H_{0}\left(\mathbf{Y}_{i+1}\right)=\mathcal{A}^{[i+1]} \otimes V^{(i+1)},
$$

where $\mathcal{M}^{[i+1]}=\mathcal{M}_{1} \otimes_{\mathcal{A}} \mathcal{M}_{2} \otimes_{\mathcal{A}} \ldots \otimes_{\mathcal{A}} \mathcal{M}_{i+1}, \mathcal{A}^{[i+1]}=\mathcal{A} /\left\langle\operatorname{det} f_{1}, \ldots, \operatorname{det} f_{i+1}\right\rangle \cong$ $\mathcal{A} /\left\langle\operatorname{det} f_{1}\right\rangle \otimes_{\mathcal{A}} \cdots \otimes_{\mathcal{A}} \mathcal{A} /\left\langle\operatorname{det} f_{i+1}\right\rangle, \mu^{[i+1]}=\mu_{1} \otimes_{\mathcal{A}} \mu_{2} \otimes_{\mathcal{A}} \cdots \otimes_{\mathcal{A}} \mu_{i+1}$, and $V^{(i+1)}=$ $V_{1} \otimes \ldots \otimes V_{i+1}$. When $p=1$ we get the commutative square

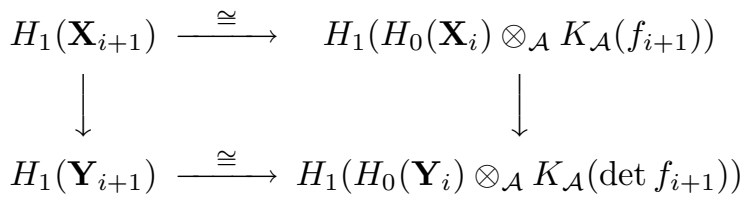

The left hand vertical arrow of the first of the above two diagrams is the 'cokernel' and the right hand vertical arrow of the second diagram is the 'kernel' of

$$
\begin{array}{lrl}
H_{0}\left(\mathbf{X}_{i}\right) \otimes_{\mathcal{A}}\left(\mathcal{A} \otimes V_{i+1}\right) \stackrel{1 \otimes_{\mathcal{A}} f_{i+1}}{\longrightarrow} & H_{0}\left(\mathbf{X}_{i}\right) \otimes_{\mathcal{A}}\left(\mathcal{A} \otimes V_{i+1}\right) \\
H_{0}\left(\mathbf{F}_{i}\right) \otimes_{\mathcal{A} 1} \downarrow & \downarrow H_{0}\left(\mathbf{F}_{i}\right) \otimes_{\mathcal{A}} \text { adj } f_{i+1} \\
H_{0}\left(\mathbf{Y}_{i}\right) \otimes_{\mathcal{A}}\left(\mathcal{A} \otimes V_{i+1}\right) \stackrel{1 \otimes_{\mathcal{A}} \operatorname{det} f_{i+1}}{\longrightarrow} & H_{0}\left(\mathbf{Y}_{i}\right) \otimes_{\mathcal{A}}\left(\mathcal{A} \otimes V_{i+1}\right)
\end{array}
$$


in which the bottom map

$$
\operatorname{det} f_{i+1}: \mathcal{A}^{[i]} \otimes V^{(i+1)} \rightarrow \mathcal{A}^{[i]} \otimes V^{(i+1)}
$$

is injective by hypothesis. If $H_{0}\left(\mathbf{F}_{i}\right)$ were also injective then we could conclude that the top map is injective, and hence also that

$$
H_{1}\left(\mathbf{X}_{i+1}\right) \stackrel{\cong}{\longrightarrow} H_{1}\left(H_{0}(\mathbf{X}) \otimes_{\mathcal{A}} K_{\mathcal{A}}\left(f_{i+1}\right)\right)=0
$$

To make the induction procedure work one must now be able to show that the 'kernel' vanishes, i.e. that $H_{0}\left(\mathbf{F}_{i+1}\right): H_{0}\left(\mathbf{X}_{i+1}\right) \rightarrow H_{0}\left(\mathbf{Y}_{i+1}\right)$ is injective.

To begin we will show that $H_{0}\left(\mathbf{F}_{i}\right) \otimes_{\mathcal{A}}$ adj $f_{i+1}$ is injective. For this it suffices to prove that

$$
1 \otimes_{\mathcal{A}} \operatorname{adj} f_{i+1}: H_{0}\left(\mathbf{Y}_{i}\right) \otimes_{\mathcal{A}}\left(\mathcal{A} \otimes V_{i+1}\right) \rightarrow H_{0}\left(\mathbf{Y}_{i}\right) \otimes_{\mathcal{A}}\left(\mathcal{A} \otimes V_{i+1}\right)
$$

is injective. The map $\operatorname{det} f_{i+1}: \mathcal{A}^{[i]} \rightarrow \mathcal{A}^{[i]}$ is injective by hypothesis. From the commutative diagram

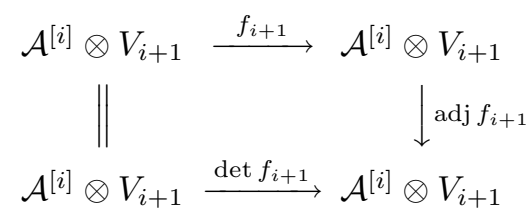

we find that $f_{i+1}: \mathcal{A}^{[i]} \otimes V_{i+1} \rightarrow \mathcal{A}^{[i]} \otimes V_{i+1}$ is injective. But the diagram

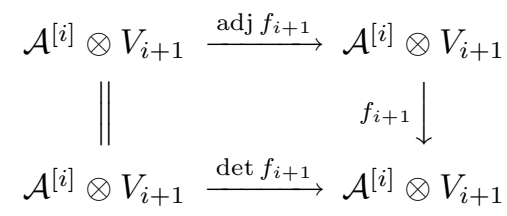

also commutes, so that

$$
\operatorname{adj} f_{i+1}: \mathcal{A}^{[i]} \otimes V_{i+1} \rightarrow \mathcal{A}^{[i]} \otimes V_{i+1}
$$

is also injective.

Let us first show that $\mu^{[i+1]}=H_{0}\left(\mathbf{F}_{i+1}\right): H_{0}\left(\mathbf{X}_{i+1}\right) \rightarrow H_{0}\left(\mathbf{Y}_{i+1}\right)$ is injective for $i=0,1$. For $i=0$ this is clear from the first diagram in this proof. The case $i=1$ is a bit more complicated. Consider the diagram

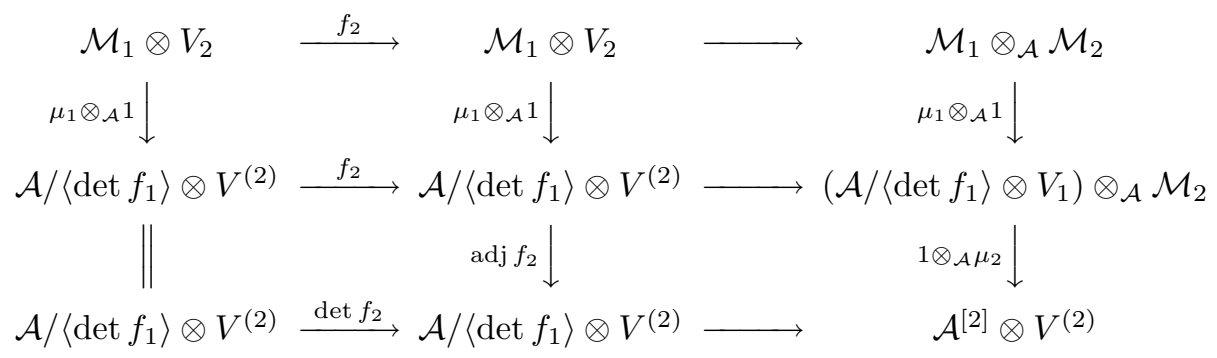

where all the vertical maps, except possibly the right hand top vertical map, are injective, the left hand horizontal maps are injective and the right hand horizontal maps are surjective. The composite of the right hand vertical maps is $H_{0}\left(\mathbf{F}_{2}\right)$. Moreover, we have the exact sequence

$$
\begin{aligned}
\operatorname{Tor}_{1}^{\mathcal{A}}\left(\mathcal{N}_{1}, \mathcal{M}_{2}\right) \rightarrow \mathcal{M}_{1} \otimes_{\mathcal{A}} \mathcal{M}_{2} & \stackrel{\mu_{1} \otimes_{\mathcal{A}} 1}{\rightarrow}\left(\mathcal{A} /\left\langle\operatorname{det} f_{1}\right\rangle \otimes V_{1}\right) \otimes_{\mathcal{A}} \mathcal{M}_{2} \\
& \rightarrow \mathcal{N}_{1} \otimes_{\mathcal{A}} \mathcal{M}_{2} \rightarrow 0
\end{aligned}
$$


and so it suffices to show that $\operatorname{Tor}_{1}^{\mathcal{A}}\left(\mathcal{N}_{1}, \mathcal{M}_{2}\right)=0$. But this follows from the commutative diagram

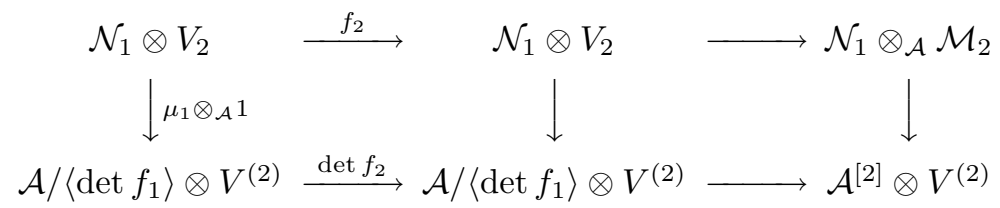

in which the left hand vertical map, the left hand bottom horizontal map and hence also the left hand top map are injective. Now let $i \geq 1$.

Induction hypothesis: For each $j$ such that $0 \leq j \leq i$ and every subset $J \subset[n]$ with $[j] \cap J=\emptyset$ and $|J|=i-j$ the map

$$
\mu^{[j]}: \mathcal{M}^{[j]} \otimes_{\mathcal{A}} \mathcal{A}^{J} \rightarrow \mathcal{A}^{[j]} \otimes_{\mathcal{A}} \mathcal{A}^{J} \otimes V^{(j)}
$$

is injective. Here $[j]=\{1,2, \ldots, j\}$ and $|J|$ is the cardinality of $J$. Furthermore, $\mathcal{A}^{J}$ is the tensor product over $\mathcal{A}$ of the quotients $\mathcal{A} /\left\langle\operatorname{det} f_{k}\right\rangle$ with $k \in J$, i.e. $\mathcal{A}^{J}=\mathcal{A} / \mathcal{J}$, where $\mathcal{J}$ is the ideal of $\mathcal{A}$ generated by $\left\{\operatorname{det} f_{k} \mid k \in J\right\}$.

If $k \notin[j] \cup J$, then by the induction hypothesis and the regularity condition the vertical maps and the bottom horizontal map in the commutative square

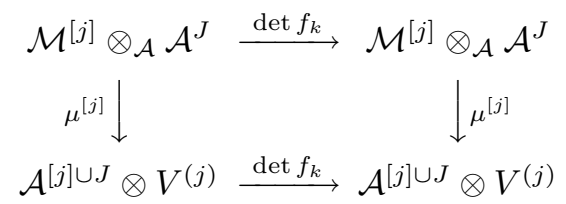

are injective, and hence so is the top horizontal map. Furthermore, since (adj $\left.f_{k}\right) f_{k}$ $=\operatorname{det} f_{k}=f_{k}\left(\operatorname{adj} f_{k}\right)$, it follows that in the commutative diagram with exact rows

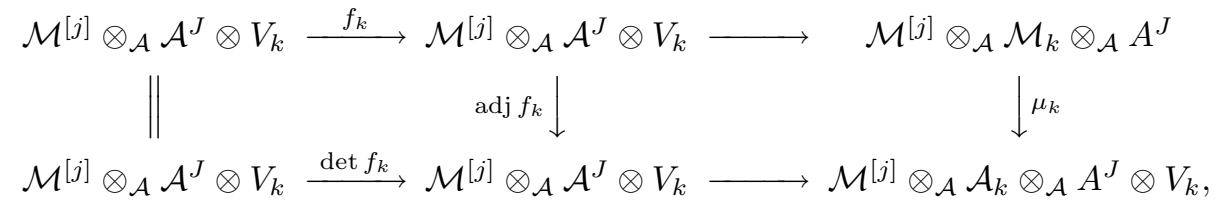

in which the right hand horizontal maps are surjective and the left hand bottom map is injective, the maps $f_{k}$ and adj $f_{k}$ are injective and hence so is $\mu_{k}$. Moreover, for every $l$ such that $1 \leq l \leq j$ and $J_{l}=(l+1, \ldots, j, k) \cup J$ it follows by the induction hypothesis and the regularity condition that the vertical maps and the bottom horizontal map in the commutative square

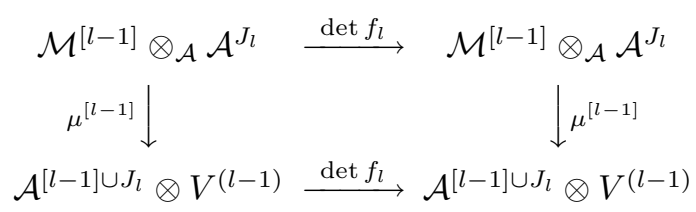

are injective, and hence so is the top horizontal map. In the commutative diagram with exact rows

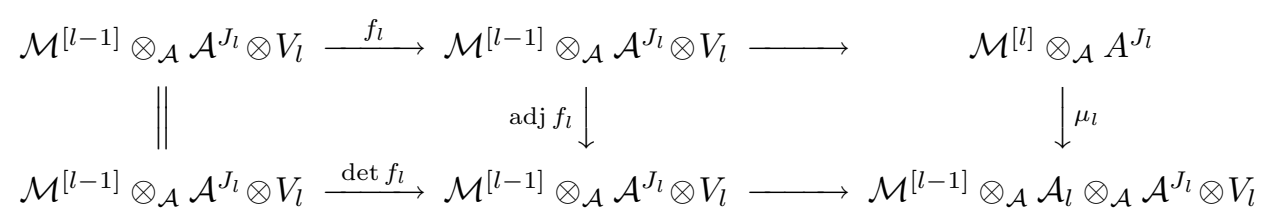


the map $\operatorname{det} f_{l}$ is injective and the right hand horizontal maps are surjective. Since $\left(\operatorname{adj} f_{l}\right) f_{l}=\operatorname{det} f_{l}=f_{l}\left(\operatorname{adj} f_{l}\right)$, it follows from the above commutative diagram that the maps $f_{l}$ and adj $f_{l}$ are also injective, and hence so is $\mu_{l}$.

Now, choosing $k=i+1$, we find by composition that

$$
\mu^{[j]}: \mathcal{M}^{[j]} \otimes_{\mathcal{A}} \mathcal{A}^{J} \rightarrow \mathcal{A}^{[j]} \otimes_{\mathcal{A}} \mathcal{A}^{J} \otimes V^{(j)}
$$

is injective for $j=0,1,2, \ldots, i+1$ and any $J \subset[n]$ with $[j] \cap J=\emptyset$ and $|J|=$ $i+1-j$.

\section{Euler Characteristic and Intersection Multiplicities}

As in the preceding sections,

$$
\operatorname{dim} \mathcal{M}=\operatorname{dim} \mathcal{A} / \operatorname{ann}_{\mathcal{A}} \mathcal{M}=\sup \{\operatorname{dim}(\mathcal{A} / \mathfrak{p}) \mid \mathfrak{p} \in \operatorname{Ass}(\mathcal{M})\}
$$

is the Krull dimension of an $\mathcal{A}$-module $\mathcal{M}$. Moreover, since $\mathcal{A}$ is a Noetherian (complete regular) local algebra over a field $F$, the 'intersection theorem'

$$
\operatorname{dim} \mathcal{N} \leq \operatorname{dh}_{\mathcal{A}} \mathcal{M}+\operatorname{dim}\left(\mathcal{M} \otimes_{\mathcal{A}} \mathcal{N}\right)
$$

of Peskine-Szpiro [4, Cor. 9.4.6] holds for finitely generated $\mathcal{A}$-modules $\mathcal{M}$ and $\mathcal{N}$.

4.1. Lemma. If $\mathbf{f}$ is regular then the $\mathcal{A}$-module $\mathcal{M}_{1} \otimes_{\mathcal{A}} \mathcal{M}_{2} \otimes_{\mathcal{A}} \cdots \otimes_{\mathcal{A}} \mathcal{M}_{n}$ has finite length, and, for $i=1,2, \ldots, n$,

1. $\operatorname{dim}\left(\mathcal{M}_{1} \otimes_{\mathcal{A}} \mathcal{M}_{2} \otimes_{\mathcal{A}} \cdots \otimes_{\mathcal{A}} \mathcal{M}_{i}\right)=n-i$,

2. $\operatorname{dim}\left(\mathcal{M}_{1} \otimes_{\mathcal{A}} \cdots \otimes_{\mathcal{A}} \mathcal{M}_{i}\right)+\operatorname{dim} \mathcal{M}_{i+1}=\operatorname{dim} \mathcal{A}+\operatorname{dim}\left(\mathcal{M}_{1} \otimes_{\mathcal{A}} \mathcal{M}_{2} \otimes_{\mathcal{A}} \cdots \otimes_{\mathcal{A}}\right.$ $\left.\mathcal{M}_{i+1}\right)$, where $\mathcal{M}_{n+1}=\mathcal{A}$,

3. $\mathcal{M}_{1} \otimes_{\mathcal{A}} \mathcal{M}_{2} \otimes_{\mathcal{A}} \cdots \otimes_{\mathcal{A}} \mathcal{M}_{i}$ is a Cohen-Macaulay module.

Proof. As in the proof of Theorem 3.3, we denote by $\mathcal{M}^{[i]}$ the tensor product $\mathcal{M}_{1} \otimes_{\mathcal{A}} \mathcal{M}_{2} \otimes_{\mathcal{A}} \cdots \otimes_{\mathcal{A}} \mathcal{M}_{i}, i=1,2, \ldots, n$. The module $\mathcal{M}^{[n]}$ is also denoted by $\mathcal{M}$. If $\mathbf{f}$ is regular then $\operatorname{det} \mathbf{f}=\left(\operatorname{det} f_{1}, \operatorname{det} f_{2}, \ldots, \operatorname{det} f_{n}\right)$ is a regular sequence in $\mathcal{A}$, so that $\mathcal{A} /\langle\operatorname{det} \mathbf{f}\rangle$ has finite length. The surjection $\mathcal{A} /\langle\operatorname{det} \mathbf{f}\rangle \otimes V \rightarrow \mathcal{M}$ implies that $\mathcal{M}$ has finite length, since $V=V_{1} \otimes V_{2} \otimes \cdots \otimes V_{n}$ is finite dimensional over $F$. By Theorem 3.3 we have the inequalities

$$
\operatorname{dh}_{\mathcal{A}} \mathcal{M}^{[i]} \leq i \text { and } \operatorname{dh}_{\mathcal{A}}\left(\mathcal{M}_{i} \otimes_{\mathcal{A}} \cdots \otimes_{\mathcal{A}} \mathcal{M}_{n}\right) \leq n-i+1
$$

for $i=1,2, \ldots, n$. Moreover, $\operatorname{dim} \mathcal{M}=0$ since $\mathcal{M}$ has finite length, and $\operatorname{dim} \mathcal{M}_{i}=$ $n-1$ by Theorem 2.1, Applying the theorem of Peskine and Szpiro [4, Thm. 9.4.5] to $\mathcal{M}=\mathcal{M}^{[i]} \otimes_{\mathcal{A}}\left(\mathcal{M}_{i+1} \otimes_{\mathcal{A}} \cdots \otimes_{\mathcal{A}} \mathcal{M}_{n}\right)$, we get that

$$
\operatorname{dim} \mathcal{M}^{[i]} \leq \operatorname{dh}_{\mathcal{A}}\left(\mathcal{M}_{i+1} \otimes_{\mathcal{A}} \cdots \otimes_{\mathcal{A}} \mathcal{M}_{n}\right) \leq n-i
$$

Now $\mathcal{M}^{[i]} \cong \mathcal{M}^{[i]} \otimes_{\mathcal{A}} \mathcal{A}$; hence [4, Cor. 9.4.6] gives

$$
n=\operatorname{dim} \mathcal{A} \leq \operatorname{dh}_{\mathcal{A}} \mathcal{M}^{[i]}+\operatorname{dim}\left(\mathcal{M}^{[i]} \otimes_{\mathcal{A}} \mathcal{A}\right) \leq i+n-i=n
$$

for $i=1,2, \ldots, n$. But then we must conclude that $\operatorname{dh}_{\mathcal{A}} \mathcal{M}^{[i]}=i$ and $\operatorname{dim} \mathcal{M}^{[i]}=$ $n-i$ for each $i$, and therefore

$$
\operatorname{dim} \mathcal{M}^{[i]}+\operatorname{dim} \mathcal{M}_{i+1}=2 n-i-1=\operatorname{dim} \mathcal{A}+\operatorname{dim} \mathcal{M}^{[i+1]}
$$

for $i=1,2, \ldots, n-1$. The equality $\operatorname{dh}_{\mathcal{A}} \mathcal{M}^{[i]}+\operatorname{codh}_{\mathcal{A}} \mathcal{M}^{[i]}=n$ [17, IV-35, Prop. 21] implies that $\operatorname{codh}_{\mathcal{A}} \mathcal{M}^{[i]}=n-i=\operatorname{dim} \mathcal{M}^{[i]}$, so that each $\mathcal{M}^{[i]}$ is a Cohen-Macaulay module. 
4.2. Corollary. If $\mathbf{q}=\left\{q_{1}, q_{2}, \ldots, q_{n}\right\}$ is a regular sequence in $\mathcal{A}$ and $p_{i}$ is a prime divisor of $q_{i}$ for each $i$, then the sequence $\mathbf{p}=\left\{p_{1}, p_{2}, \ldots, p_{n}\right\}$ is also regular.

Proof. Since $\mathcal{A} /\left\langle q_{1}, \ldots, q_{n}\right\rangle$ has finite length, also $\mathcal{A} /\left\langle p_{1}, \ldots, p_{n}\right\rangle$ has finite length, and $\operatorname{dim} \mathcal{A} /\left\langle p_{i}\right\rangle=n-1$. Observe that Theorem 3.3 is still valid if we replace $\mathcal{M}_{i}$ by $\mathcal{A} /\left\langle p_{i}\right\rangle, f_{i}$ by $p_{i}$, adj $f_{i}$ by $q_{i} / p_{i}$, and $\operatorname{det} f_{i}$ by $q_{i}$. Now taking $\mathcal{M}_{i}=\mathcal{A} /\left\langle p_{i}\right\rangle$ in Lemma 4.1, we see that $\mathbf{p}$ is a regular sequence.

Next we recall some of the definitions and the properties of the theory of cycles [17]. If $\mathfrak{q}$ is a prime ideal of $\mathcal{A}$, then $\mathrm{ht} \mathfrak{q}=\operatorname{dim} \mathcal{A}_{\mathfrak{q}}$ and $\operatorname{coht} \mathfrak{q}=\operatorname{dim} \mathcal{A} / \mathfrak{q}$. Moreover, ht $\mathfrak{q}+\operatorname{coht} \mathfrak{q}=\operatorname{dim} \mathcal{A}=n$, since $\mathcal{A}$ is a finitely generated $F$-algebra and an integral domain. The free abelian group

$$
Z(\mathcal{A})=\left\{\sum z(\mathfrak{q}) \mathfrak{q} \mid z(\mathfrak{q}) \in \mathbb{Z}\right\}
$$

generated by $\operatorname{Spec}(\mathcal{A})$ is the group of cycles of $\mathcal{A}$. If

$$
Z_{a}(\mathcal{A})=\left\{\sum_{\operatorname{coht} \mathfrak{q}=a} z(\mathfrak{q}) \mathfrak{q} \mid z(\mathfrak{q}) \in \mathbb{Z}\right\}
$$

is the subgroup of cycles of coheight $a$, then $Z(\mathcal{A})=\bigoplus Z_{a}(\mathcal{A})$. A cycle $z=\sum z(\mathfrak{q}) \mathfrak{q}$ is called positive if and only if $z(\mathfrak{q}) \geq 0$ for all $\mathfrak{q}$. Grading by height or codimension gives $Z^{\alpha}(\mathcal{A})=Z_{n-\alpha}(\mathcal{A})=\left\{\sum_{\mathrm{ht} \mathfrak{q}=\alpha} z(\mathfrak{q}) \mathfrak{q} \mid z(\mathfrak{q}) \in \mathbb{Z}\right\}$.

If $\operatorname{dim} \mathcal{A}=n$ and $a+b=n+c$, then the cycle product

$$
\cdot: Z_{a}(\mathcal{A}) \otimes Z_{b}(\mathcal{A}) \rightarrow Z_{c}(\mathcal{A})
$$

is defined by linearity:

$$
z_{a} \cdot z_{b}=\sum_{\text {coht } \mathfrak{r}=c} z_{a}(\mathfrak{p}) z_{b}(\mathfrak{q}) \chi^{\mathcal{A}_{\mathfrak{r}}}\left(\mathcal{A}_{\mathfrak{r}} / \mathfrak{p}, \mathcal{A}_{\mathfrak{r}} / \mathfrak{q}\right) \mathfrak{r}
$$

i.e. $\mathfrak{p} \cdot \mathfrak{q}=\sum_{\text {coht } \mathfrak{r}=c} \chi^{\mathcal{A}_{\mathfrak{r}}}\left(\mathcal{A}_{\mathfrak{r}} / \mathfrak{p}, \mathcal{A}_{\mathfrak{r}} / \mathfrak{q}\right) \mathfrak{r}$. Grading by height or codimension gives

$$
\cdot: Z^{\alpha}(\mathcal{A}) \otimes Z^{\beta}(\mathcal{A}) \rightarrow Z^{\alpha+\beta}(\mathcal{A}),
$$

where

$$
z^{\alpha} \cdot z^{\beta}=\sum_{\text {ht } \mathfrak{r}=\alpha+\beta} z^{\alpha}(\mathfrak{p}) z^{\beta}(\mathfrak{q}) \chi^{\mathcal{A}_{\mathfrak{r}}}\left(\mathcal{A}_{\mathfrak{r}} / \mathfrak{p}, \mathcal{A}_{\mathfrak{r}} / \mathfrak{q}\right) \mathfrak{r}
$$

i.e. $\mathfrak{p} \cdot \mathfrak{q}=\sum_{\mathrm{ht} \mathfrak{r}=\alpha+\beta} \chi^{\mathcal{A}_{\mathfrak{r}}}\left(\mathcal{A}_{\mathfrak{r}} / \mathfrak{p}, \mathcal{A}_{\mathfrak{r}} / \mathfrak{q}\right) \mathfrak{r}$.

For any finitely generated $\mathcal{A}$-module $\mathcal{M}$ and every integer $a$, consider the cycle $[17, \mathrm{~V}-1-2]$

$$
z_{a}(\mathcal{M})=\sum_{\operatorname{coht} \mathfrak{q}=a} l_{\mathfrak{q}}(\mathcal{M}) \mathfrak{q},
$$

where $l_{\mathfrak{q}}(\mathcal{M})=l^{\mathcal{A}_{\mathfrak{q}}}\left(\mathcal{M}_{\mathfrak{q}}\right)$, coht $q=\operatorname{dim} \mathcal{A} / \mathfrak{q}$, and the sum is over all prime ideals $\mathfrak{q}$ of coheight $a$. If $\operatorname{dim} \mathcal{M} \leq a, \operatorname{dim} \mathcal{N} \leq b$ and $\operatorname{dim}\left(\mathcal{M} \otimes_{\mathcal{A}} \mathcal{N}\right) \leq c$ with $a+b=n+c$, then the cycles $z_{a}(\mathcal{M})$ and $z_{b}(\mathcal{N})$ are defined and intersect properly, and Serre's theorem $[17, \mathrm{~V}-22]$ says that the intersection cycle is given by

$$
z_{a}(\mathcal{M}) \cdot z_{b}(\mathcal{N})=z_{c}\left(\operatorname{Tor}^{\mathcal{A}}(\mathcal{M}, \mathcal{N})\right)=\sum_{i}(-1)^{i} z_{c}\left(\operatorname{Tor}_{i}^{\mathcal{A}}(\mathcal{M}, \mathcal{N})\right)
$$


where the coefficient of a prime ideal $\mathfrak{p} \in V\left(\mathcal{M} \otimes_{\mathcal{A}} \mathcal{N}\right)$ with coht $\mathfrak{p}=c$ is the Euler characteristic

$$
\chi_{\mathfrak{p}}(\mathcal{M}, \mathcal{N})=\sum_{i}(-1)^{i} l\left(\operatorname{Tor}_{i}^{\mathcal{A}}(\mathcal{M}, \mathcal{N})_{\mathfrak{p}}\right)
$$

which is additive in $\mathcal{M}$ and $\mathcal{N}$.

Some of the properties of the cycle function $z_{a}$ are:

1. $z_{a}(\mathcal{M}) \geq 0$.

2. $z_{a}(\mathcal{M})=0$ if $\operatorname{dim} \mathcal{M} \leq a-1$ : If $\operatorname{dim} \mathcal{A} / \operatorname{ann} \mathcal{M}=\operatorname{dim} \mathcal{M} \leq a-1$ and coht $q=a$, then ann $\mathcal{M}$ is not in $q$ and hence $\mathcal{M}_{q}=0$.

3. $z_{a}$ is additive: If $0 \rightarrow \mathcal{N} \rightarrow \mathcal{M} \rightarrow \mathcal{L} \rightarrow 0$ is exact then so is $0 \rightarrow \mathcal{N}_{q} \rightarrow \mathcal{M}_{q} \rightarrow$ $\mathcal{L}_{q} \rightarrow 0$. Hence, $l\left(\mathcal{M}_{q}\right)=l\left(\mathcal{N}_{q}\right)+l\left(\mathcal{L}_{q}\right)$, so that $z_{a}(\mathcal{M})=z_{a}(\mathcal{N})+z_{a}(\mathcal{L})$.

4. Universal property: Every additive function on $K_{a}(\mathcal{A})$ taking positive values in an ordered abelian group factors through $z_{a}$.

5. If $\mathcal{A}$ is a domain and $\operatorname{dim} \mathcal{A}=n$, then $Z_{n}(\mathcal{A}) \cong \mathbb{Z}$ and $z_{n}: K_{n}(\mathcal{A}) \rightarrow \mathbb{Z}$ is the rank function.

4.3. Theorem. Let $\mathbf{f}$ be an n-parameter system, $\mathfrak{m} \in \sigma(\mathbf{f})$, and let $I$ be the ideal $\left\langle\operatorname{det} f_{1}, \operatorname{det} f_{2}, \ldots, \operatorname{det} f_{n}\right\rangle$ in $\mathcal{A}=A_{\mathfrak{m}}$. If $\mathbf{f}$ is regular at $\mathfrak{m}$, then $\mathcal{M}=\mathcal{M}_{1} \otimes_{\mathcal{A}}$ $\mathcal{M}_{2} \otimes_{\mathcal{A}} \ldots \otimes_{\mathcal{A}} \mathcal{M}_{n}$ has finite length and

$l^{\mathcal{A}}(\mathcal{M})=\sum_{p_{i} \mid \operatorname{det} f_{i}} l_{p_{1}}\left(\mathcal{M}_{1}\right) \ldots l_{p_{n}}\left(\mathcal{M}_{n}\right) l^{\mathcal{A}}\left(\mathcal{A} /\left\langle p_{1}\right\rangle, \mathcal{A} /\left\langle p_{2}\right\rangle, \ldots, \mathcal{A} /\left\langle p_{n}\right\rangle\right)=l^{\mathcal{A}}(\mathcal{A} / I)$,

where the length $l_{p_{i}}\left(\mathcal{M}_{i}\right)$ of the localization of $\mathcal{M}_{i}$ at $p_{i}$ is equal to the multiplicity of $p_{i}$ in $\operatorname{det} f_{i}$ and $l^{\mathcal{A}}\left(\mathcal{A} /\left\langle p_{1}\right\rangle, \mathcal{A} /\left\langle p_{2}\right\rangle, \ldots, \mathcal{A} /\left\langle p_{n}\right\rangle\right)=\chi^{\mathcal{A}}\left(\mathcal{A} /\left\langle p_{1}, p_{2}, \ldots, p_{n}\right\rangle\right)=$ $i\left(p_{1}, p_{2}, \ldots, p_{n}\right)$ is the intersection multiplicity of the hypersurfaces defined by the irreducible polynomials $p_{i}$ at the point $\mathfrak{m}$.

Proof. Lemma 4.1 says that

$$
n-s=\operatorname{dim}\left(\mathcal{M}_{1} \otimes_{\mathcal{A}} \ldots \otimes_{\mathcal{A}} \mathcal{M}_{s}\right)=\sup \left\{\operatorname{coht} \mathfrak{p} \mid \mathfrak{p} \in \operatorname{Ass}\left(\mathcal{M}_{1} \otimes_{\mathcal{A}} \ldots \otimes_{\mathcal{A}} \mathcal{M}_{s}\right)\right\} .
$$

In particular, $\mathcal{M}$ has finite length and the cycles $z_{n-1}\left(\mathcal{M}_{i}\right)$ intersect properly. In fact, since $\operatorname{Tor}^{\mathcal{A}}\left(\mathcal{M}_{1} \otimes_{\mathcal{A}} \ldots \otimes_{\mathcal{A}} \mathcal{M}_{s}, \mathcal{M}_{s+1}\right)=\mathcal{M}_{1} \otimes_{\mathcal{A}} \ldots \otimes_{\mathcal{A}} \mathcal{M}_{s+1}$ by Theorem $3.3(2)$ and

$$
\operatorname{dim}\left(\mathcal{M}_{1} \otimes_{\mathcal{A}} \cdots \otimes_{\mathcal{A}} \mathcal{M}_{s}\right)+\operatorname{dim} \mathcal{M}_{s+1}=\operatorname{dim} \mathcal{A}+\operatorname{dim}\left(\mathcal{M}_{1} \otimes_{\mathcal{A}} \cdots \otimes_{\mathcal{A}} \mathcal{M}_{s+1}\right)
$$

by Lemma 4.1 , the cycles $z_{n-s}\left(\mathcal{M}_{1} \otimes_{\mathcal{A}} \ldots \otimes_{\mathcal{A}} \mathcal{M}_{s}\right)$ and $z_{n-1}\left(\mathcal{M}_{s+1}\right)$ intersect properly for each $s=1,2, \ldots, n-1[17, \mathrm{p}$. V-22, Prop. 1]. Now one proves by induction on $s$ that

$$
z_{n-1}\left(\mathcal{M}_{1}\right) \cdot z_{n-1}\left(\mathcal{M}_{2}\right) \cdot \ldots \cdot z_{n-1}\left(\mathcal{M}_{s}\right)=z_{n-s}\left(\mathcal{M}_{1} \otimes_{\mathcal{A}} \ldots \otimes_{\mathcal{A}} \mathcal{M}_{s}\right)
$$

In particular, for $s=n$ we get

$$
z_{n-1}\left(\mathcal{M}_{1}\right) \cdot z_{n-1}\left(\mathcal{M}_{2}\right) \cdot \ldots \cdot z_{n-1}\left(\mathcal{M}_{n}\right)=z_{0}(\mathcal{M})=l^{\mathcal{A}}(\mathcal{M}) \mathfrak{m} .
$$

By Theorem 2.1 the prime ideals of coheight $n-1$ in $\operatorname{Var}\left(\mathcal{M}_{i}\right)$ are exactly the principal primes generated by the prime divisors of $\operatorname{det} f_{i}$, and so

$$
z_{n-1}\left(\mathcal{M}_{i}\right)=\sum_{\operatorname{coht} \mathfrak{q}=n-1} l_{\mathfrak{q}}\left(\mathcal{M}_{i}\right) \mathfrak{q}=\sum_{p \mid \operatorname{det} f_{i}} l_{\langle p\rangle}\left(\mathcal{M}_{i}\right)\langle p\rangle,
$$


where $p$ runs over all prime divisors of $\operatorname{det} f_{i}$. The associativity of the cycle product shows that the coefficient of $\mathfrak{m}$ on the left hand side of the equation is exactly

$$
\sum_{p_{i} \mid \operatorname{det} f_{i}} l_{p_{1}}\left(\mathcal{M}_{1}\right) \ldots l_{p_{n}}\left(\mathcal{M}_{n}\right) \chi\left(\mathcal{A} /\left\langle p_{1}\right\rangle, \ldots, \mathcal{A} /\left\langle p_{n}\right\rangle\right) .
$$

The same procedure applied to the system $\operatorname{det} \mathbf{f}$ shows that this is also equal to $l(\mathcal{A} /\langle\operatorname{det} \mathbf{f}\rangle)$. Furthermore, it follows by Corollary 4.2 that we may replace the Euler characteristic $\chi^{\mathcal{A}}\left(\mathcal{A} /\left\langle p_{1}\right\rangle, \ldots, \mathcal{A} /\left\langle p_{n}\right\rangle\right)$ by the length $l^{\mathcal{A}}\left(\mathcal{A} /\left\langle p_{1}, \ldots, p_{n}\right\rangle\right)$.

If the Koszul complex $K_{A}(\mathbf{f})$ of the $n$-parameter system $\mathbf{f}$ on the affine space $\mathbb{A}^{n}$ is acyclic, then so is $K_{\mathcal{A}}(\mathbf{f})$ at every point $\boldsymbol{\lambda}$, since localization and completion are exact. In this case the assertions of Theorem 4.3 hold for every point $\lambda$ in $\mathbb{A}^{n}$, i.e. at the ideal $\mathfrak{m}$ generated by the polynomials $x_{i}-\lambda_{i}, i=1,2, \ldots, n$.

Note that if the residue class field $\mathcal{A} / \mathfrak{m} \mathcal{A}$ of $\mathcal{A}$ is isomorphic to the base field $F$, then the length of the $\mathcal{A}$-module $\mathcal{M}$ coincides with the (vector space) dimension of $\mathcal{M}$ over $F$, i.e. $l^{\mathcal{A}}(\mathcal{M})=\operatorname{dim}_{F}(\mathcal{M})$. Since $\mathcal{M}$ is finite dimensional, there is a positive integer, and hence a least positive integer $r$, such that $\mathfrak{m}^{r} \mathcal{M}=0$, i.e. $\mathfrak{m}^{r} \subset \operatorname{ann}_{\mathcal{A}} \mathcal{M}$. On the other hand, since the $\operatorname{det} f_{i}$ form a regular sequence, there is an integer, hence a least integer $s$, such that $\mathfrak{m}^{s} \subset I$, i.e. $\operatorname{ann}_{\mathcal{M}} \mathfrak{m}^{s}=\mathcal{M}$ (see $[18$, p. 186, Lemma 1]). The string of inclusions

$$
I \subseteq \sum_{j=1}^{n} \operatorname{ann}_{\mathcal{A}} \mathcal{M}_{j} \subseteq \operatorname{ann}_{\mathcal{A}} \mathcal{M} \subset \mathfrak{m}
$$

implies that $r \leq s$, with equality if $I=\operatorname{ann}_{\mathcal{A}} \mathcal{M}$.

Observe that Theorem 4.3 generalizes a result of Binding and Browne [3], who proved for a particular class of linear two-parameter systems with $F=\mathbb{R}$ that the dimension of the root subspace (in our notation, of $\mathcal{M}_{1} \otimes_{\mathcal{A}} \mathcal{M}_{2}$ ) at the simple point $\lambda$ equals the sum of the orders of contact of the eigencurves (counting multiplicities) passing through $\boldsymbol{\lambda}$. For the two-parameter case and also for a simple point in the spectrum we have a more direct proof of Theorem 4.3 avoiding the use of Theorem 3.3 .

4.4. Theorem. If the 2-parameter system $\mathbf{f}$ is regular at the point $\mathfrak{m} \in \sigma(\mathbf{f})$, then $\mathcal{M}_{1} \otimes_{\mathcal{A}} \mathcal{M}_{2}$ has finite length and

$l^{\mathcal{A}}\left(\mathcal{M}_{1} \otimes_{\mathcal{A}} \mathcal{M}_{2}\right)=\sum_{p_{i} \mid \operatorname{det} f_{i}} l_{p_{1}}\left(\mathcal{M}_{1}\right) l_{p_{2}}\left(\mathcal{M}_{2}\right) l^{\mathcal{A}}\left(\mathcal{A} /\left\langle p_{1}, p_{2}\right\rangle\right)=l^{\mathcal{A}}\left(\mathcal{A} /\left\langle\operatorname{det} f_{1}, \operatorname{det} f_{2}\right\rangle\right)$,

where

$$
l^{\mathcal{A}}\left(\mathcal{A} /\left\langle p_{1}, p_{2}\right\rangle\right)=i\left(p_{1}, p_{2}\right)
$$

is the intersection multiplicity of the algebraic curves defined by the irreducible polynomials $p_{1}$ and $p_{2}$ at the point $\mathfrak{m}$.

Proof. By 2.1 there are canonical surjective $\mathcal{A}$-module maps $\pi_{i}: \mathcal{A} /\left\langle\operatorname{det} f_{i}\right\rangle \otimes V_{i} \rightarrow$ $\mathcal{M}_{i}$ for each $i=1,2$, and hence a surjection of $\mathcal{A}$-modules $\mathcal{A} / I \otimes V \rightarrow \mathcal{M}$, where $I$ is the ideal $\left\langle\operatorname{det} f_{1}, \operatorname{det} f_{2}\right\rangle \subset \mathcal{A}$ and $\mathcal{M}=\mathcal{M}_{1} \otimes_{\mathcal{A}} \mathcal{M}_{2}$. The quotient $\mathcal{A} / I$ has finite length, i.e. $\operatorname{Ass} \mathcal{A} / I=\{\mathfrak{m}\}$, since $K_{\mathcal{A}}(\operatorname{det} \mathbf{f})$ is acyclic. Now $\mathcal{M}$ has finite length, since $\mathcal{A} / I$ does and since $V$ is finite-dimensional. It now suffices to observe that $\operatorname{dim} \mathcal{A}=2$ and $\operatorname{dim} \mathcal{M}_{i}=1$ for $i=1,2$. Since $\bigcup_{\mathfrak{p} \in \operatorname{Ass} \mathcal{M}_{i}} \mathfrak{p}$ is the set of zerodivisors for $\mathcal{M}_{i}$ in $\mathcal{A}$ and $\mathfrak{m} \notin \operatorname{Ass} \mathcal{M}_{i}$ there is a non-zerodivisor $a_{i} \in \mathfrak{m}$ for $\mathcal{M}_{i}$; 
hence $\operatorname{dim} \mathcal{M}_{i} / a_{i} \mathcal{M}_{i}=0$. This means that $\operatorname{codh} \mathcal{M}_{i}=\operatorname{dim} \mathcal{M}_{i}$, i.e. that $\mathcal{M}_{i}$ is a Cohen-Macaulay $\mathcal{A}$-module, and we conclude by [17, V-19] that $\operatorname{Tor}_{k}^{\mathcal{A}}\left(\mathcal{M}_{1}, \mathcal{M}_{2}\right)=0$ for $k>0$. This implies that $\chi_{\mathfrak{m}}\left(\operatorname{Tor}^{\mathcal{A}}\left(\mathcal{M}_{1}, \mathcal{M}_{2}\right)\right)=l^{\mathcal{A}}\left(\mathcal{M}_{1} \otimes_{\mathcal{A}} \mathcal{M}_{2}\right)$. For the rest see $\left[8,18\right.$ p.186], or prove it directly as follows. The pair $\left(\operatorname{det} f_{1}, \operatorname{det} f_{2}\right)$ forms a regular sequence in $\mathcal{A}$, and so $\operatorname{det} f_{1}$ and $\operatorname{det} f_{2}$ must be relatively prime. Moreover, every pair of irreducible divisors $\left\{p_{1}, p_{2}\right\}$ of $\left\{\operatorname{det} f_{1}, \operatorname{det} f_{2}\right\}$ forms a regular sequence in the Noetherian regular local (hence Cohen-Macaulay) algebra $\mathcal{A}$ [18, p. 40, 18]. It follows by [17, IV-5] that the Koszul complex $K_{\mathcal{A}}\left(p_{1}, p_{2}\right) \cong K_{\mathcal{A}}\left(p_{1}\right) \otimes_{\mathcal{A}} K_{\mathcal{A}}\left(p_{2}\right)$ is a free resolution of the $\mathcal{A}$-module $\mathcal{A} /\left\langle p_{1}, p_{2}\right\rangle$. We conclude that

$$
\operatorname{Tor}^{\mathcal{A}}\left(\mathcal{A} /\left\langle p_{1}\right\rangle, \mathcal{A} /\left\langle p_{2}\right\rangle\right)=\mathcal{A} /\left\langle p_{1}\right\rangle \otimes_{\mathcal{A}} \mathcal{A} /\left\langle p_{2}\right\rangle \cong \mathcal{A} /\left\langle p_{1}, p_{2}\right\rangle
$$

by $[17, \mathrm{~V}-26]$, so that $\chi^{\mathcal{A}}\left(\mathcal{A} /\left\langle p_{1}\right\rangle, \mathcal{A} /\left\langle p_{2}\right\rangle\right)=l^{\mathcal{A}}\left(\mathcal{A} /\left\langle p_{1}, p_{2}\right\rangle\right)$.

4.5. Theorem. If $\mathfrak{m}$ is a simple regular point for the multiparameter system $\mathbf{f}$, then

$$
\mathcal{M} \cong \mathcal{A} /\left\langle\operatorname{det} f_{1}, \ldots, \operatorname{det} f_{n}\right\rangle
$$

and

$$
l^{\mathcal{A}}(\mathcal{M})=\sum_{p_{i} \mid \operatorname{det} f_{i}} l_{p_{1}}\left(\mathcal{M}_{1}\right) \ldots i_{p_{n}}\left(M_{n}\right) l^{\mathcal{A}}\left(\mathcal{A} /\left\langle p_{1}, p_{2}, \ldots, p_{n}\right\rangle\right)=l^{\mathcal{A}}(\mathcal{A} / I)
$$

where $I=\left\langle\operatorname{det} f_{1}, \operatorname{det} f_{2}, \ldots, \operatorname{det} f_{n}\right\rangle$ and $l^{\mathcal{A}}\left(\mathcal{A} /\left\langle p_{1}, p_{2}, \ldots, p_{n}\right\rangle=i\left(p_{1}, p_{2}, \ldots, p_{n}\right)\right.$ is the intersection multiplicity of the hypersurfaces defined by the irreducible polynomials $p_{i}$ at the point $\mathfrak{m}$.

Proof. It suffices to show that the Koszul complex $K_{\mathcal{A}}(\mathbf{f})$ is acyclic. The Koszul complex $K_{\mathcal{A}}(\operatorname{det} \mathbf{f})$ is acyclic by assumption. It follows in particular that $\operatorname{det} f_{i} \neq 0$, and hence that $f_{i}: \mathcal{A} \otimes V_{i} \rightarrow \mathcal{A} \otimes V_{i}$ is injective for $i=1,2, \ldots, n$, so that $K_{\mathcal{A}}\left(f_{i}\right)$ is a free resolution of the $\mathcal{A}$-module $\mathcal{M}_{i}$. By Theorem 2.2 we have that $\mathcal{M}_{i} \cong \mathcal{A} /\left\langle\operatorname{det} f_{i}\right\rangle$. Now we prove by induction that $K_{\mathcal{A}}(\mathbf{f})$ is acyclic. Assume that $Y_{i}=K_{\mathcal{A}}\left(f_{1}\right) \otimes_{\mathcal{A}} K_{\mathcal{A}}\left(f_{2}\right) \otimes_{\mathcal{A}} \ldots \otimes_{\mathcal{A}} K_{\mathcal{A}}\left(f_{i}\right)$ is a free resolution of $\mathcal{M}_{1} \otimes_{\mathcal{A}} \mathcal{M}_{2} \otimes_{\mathcal{A}}$ $\ldots \otimes_{\mathcal{A}} \mathcal{M}_{i} \cong \mathcal{A} /\left\langle\operatorname{det} f_{1}, \operatorname{det} f_{2}, \ldots, \operatorname{det} f_{i}\right\rangle$. The acyclicity of $K_{\mathcal{A}}(\operatorname{det} \mathbf{f})$ implies that

$$
\operatorname{Tor}_{j}^{\mathcal{A}}\left(\mathcal{A} /\left\langle\operatorname{det} f_{1}, \operatorname{det} f_{2}, \ldots, \operatorname{det} f_{i}\right\rangle, \mathcal{A} /\left\langle\operatorname{det} f_{i+1}\right\rangle\right)=0
$$

for $j \neq 0$, and therefore that

$$
H_{j}^{\mathcal{A}}\left(Y_{i+1}\right)=\operatorname{Tor}_{j}^{\mathcal{A}}\left(\mathcal{M}_{1} \otimes_{\mathcal{A}} \mathcal{M}_{2} \otimes_{\mathcal{A}} \ldots \otimes_{\mathcal{A}} \mathcal{M}_{i}, \mathcal{M}_{i+1}\right)=0
$$

for $j \neq 0$. We conclude that $Y_{i+1}$ is acyclic, and the proof is complete when $i=n-1$.

\section{The Linear Multiparameter Case}

A linear n-parameter system $\mathbf{f}$ is a system of linear polynomials

$$
f_{i}(\mathbf{x})=\sum_{j=1}^{n} A_{i j} x_{j}-A_{i 0} \quad(i=1,2, \ldots, n)
$$

in $n$ variables $\mathbf{x}=\left(x_{1}, x_{2}, \ldots, x_{n}\right)$, where we assume that the coefficients $A_{i j}$ are linear maps acting on a finite-dimensional vector space $V_{i}$ over a field $F$. From now on we assume that $F$ is infinite. The linear map $A_{i j}$ induces a linear map $A_{i j}^{\dagger}$ on 
the vector space $V=V_{1} \otimes V_{2} \otimes \cdots \otimes V_{n}$ by acting on the $i$-th tensor factor. The determinant $\Delta_{0}$ of the matrix

$$
\left(\begin{array}{cccc}
A_{11}^{\dagger} & A_{12}^{\dagger} & \cdots & A_{1 n}^{\dagger} \\
A_{21}^{\dagger} & A_{22}^{\dagger} & \cdots & A_{2 n}^{\dagger} \\
\vdots & \vdots & & \vdots \\
A_{n 1}^{\dagger} & A_{n 2}^{\dagger} & \cdots & A_{n n}^{\dagger}
\end{array}\right)
$$

is a linear transformation on $V$. It is well defined because any two entries from distinct rows in the above matrix commute. In a similar way linear transformations $\Delta_{i}(i=1,2, \ldots, n)$ on $V$ are defined by replacing the $i$-th column in the matrix by $\left[A_{k 0}^{\dagger}\right]_{k=1}^{n}$.

Recall that for each $f_{i}$ we have the short exact sequence

$$
F[\mathbf{x}] \otimes V_{i} \stackrel{f_{i}}{\longrightarrow} F[\mathbf{x}] \otimes V_{i} \rightarrow M_{i} \rightarrow 0
$$

and its localization at a maximal ideal $m$ in the variety $\operatorname{Var} M_{i}$

$$
\mathcal{A} \otimes V_{i} \stackrel{f_{i}}{\longrightarrow} \mathcal{A} \otimes V_{i} \rightarrow \mathcal{M}_{i} \rightarrow 0 .
$$

5.1. Theorem. For a linear multiparameter system $\mathbf{f}$ over an infinite field $F$ the following are equivalent:

1. $\mathbf{f}$ is regular, i.e. $K_{A}(\operatorname{det} \mathbf{f})$ is acyclic,

2. there exist $\alpha_{i} \in F(i=0,1, \ldots, n)$ such that $\sum_{i=0}^{n} \alpha_{i} \Delta_{i}$ is invertible,

3. the spectrum $\sigma(\mathbf{f})$ is finite,

4. the (Krull) dimension $\operatorname{dim} F[\mathbf{x}] /\left\langle\operatorname{det} f_{1}, \operatorname{det} f_{2}, \ldots, \operatorname{det} f_{n}\right\rangle=0$,

5. the sequence $\left(\operatorname{det} f_{1}, \operatorname{det} f_{2}, \ldots, \operatorname{det} f_{n}\right)$ is a regular sequence in $F[\mathbf{x}]$.

Each of the above statements implies:

6. the Koszul complex $K_{\mathcal{A}}(\mathbf{f})$ is acyclic for all $m \in \sigma(\mathbf{f})$, and $H_{\mathcal{A}}^{0}(\mathbf{f})$ has finite length.

Proof. The implication (2) $\Rightarrow(3)$ was proved by Atkinson in [2, Thm. 6.8.1], and the inverse implication $(3) \Rightarrow(2)$ follows from [2, Thm. 8.7.2]. (Note that in the proof of [2, Thms. 8.2.1 and 8.7.2] the fact that $F$ is an infinite field is used. This is the case, for instance, if $\operatorname{char} F=0$.) To show that $(3) \Leftrightarrow(4)$ note that the spectrum $\sigma(\mathbf{f})$ coincides with the set of all maximal ideals containing $\operatorname{det} \mathbf{f}$. This set is finite if and only if $\operatorname{dim} F[\mathbf{x}] /\left\langle\operatorname{det} f_{1}, \operatorname{det} f_{2}, \ldots, \operatorname{det} f_{n}\right\rangle=0$. The equivalencies $(1) \Leftrightarrow(4)$ and $(1) \Leftrightarrow(5)$ are proved by Serre in [17, p. III-11, Prop. 6] and [17, p. IV-5, Prop. 3], respectively.

Finally it follows by Theorem 3.3 that $(1) \Rightarrow(6)$.

Each of the equivalent statements (1), (3), (4) or (5) could be used as a definition of regularity of a linear multiparameter system. Theorem 5.1 implies that in the linear case our definition of regularity is equivalent with the standard one, i.e. to the statement (2) in Theorem 5.1 (see e.g. [2]).

The following is a restatement of Theorem 4.3 for the linear case.

5.2. Theorem. If $\mathbf{f}$ is a regular linear n-parameter system on the affine space $\mathbb{A}^{n}$ then the $\mathcal{A}$-module $\mathcal{M}=\mathcal{M}_{1} \otimes_{\mathcal{A}} \mathcal{M}_{2} \otimes_{\mathcal{A}} \ldots \otimes_{\mathcal{A}} \mathcal{M}_{n}$ has finite length and

$$
l^{\mathcal{A}}\left(\mathcal{M}_{1} \otimes_{\mathcal{A}} \ldots \otimes_{\mathcal{A}} \mathcal{M}_{n}\right)=\sum_{p_{i} \mid \operatorname{det} f_{i}} l_{p_{1}}\left(\mathcal{M}_{1}\right) \ldots l_{p_{n}}\left(\mathcal{M}_{n}\right) l^{\mathcal{A}}\left(\mathcal{A} /\left\langle p_{1}, \ldots, p_{n}\right\rangle\right),
$$


where

$$
l^{\mathcal{A}}\left(\mathcal{A} /\left\langle p_{1}, p_{2}, \ldots, p_{n}\right\rangle\right)=i\left(p_{1}, p_{2}, \ldots, p_{n}\right)
$$

is the intersection multiplicity of the hypersurfaces defined by the irreducible polynomials $p_{i}$ at the point $m \in \sigma(\mathbf{f})$.

5.3. Example. Let us consider the linear 2-parameter system $\mathbf{f}$, where the matrices $f_{1}(\mathbf{x})$ and $f_{2}(\mathbf{x})$ are given by

$$
\left(\begin{array}{cccc}
x_{1}+x_{2}+1 & 0 & 0 & 0 \\
2 & 2 x_{1}+x_{2}+1 & 1-x_{2} & 2 \\
0 & x_{1}+x_{2} & x_{1}+x_{2} & x_{2}-1 \\
0 & 0 & 0 & x_{1}+2 x_{2}
\end{array}\right) \text { and }\left(\begin{array}{ccc}
x_{2}-1 & x_{1}-1 & 0 \\
x_{1} & x_{1} & 0 \\
0 & 1 & x_{2}
\end{array}\right)
$$

This system is simple at the point $m=\left\langle x_{1}, x_{2}\right\rangle$, i.e. the joint eigenspace at $\boldsymbol{\lambda}=$ $(0,0)$ is 1 -dimensional, so by Corollary 4.5 we have

$$
\mathcal{M}=\mathcal{M}_{1} \otimes_{A} \mathcal{M}_{2} \cong \mathcal{A} /\left\langle\operatorname{det} f_{1}, \operatorname{det} f_{2}\right\rangle,
$$

where $\operatorname{det} f_{1}=2\left(x_{1}+x_{2}+1\right)\left(x_{1}+2 x_{2}\right)\left(x_{1}+x_{2}\right)^{2}$ and $\operatorname{det} f_{2}=x_{1}\left(x_{2}-x_{1}\right) x_{2}$. A direct calculation shows that for $i=1,2, \ldots$ the lengths of the filtered modules $\mathcal{M} / m^{i} \mathcal{M}$ are $1,3,6,8,9,9, \ldots$ respectively, and thus $l^{\mathcal{A}}(\mathcal{M})=\operatorname{dim}_{F} \mathcal{M}=9$. Let $p_{11}=x_{1}+2 x_{2}, p_{12}=x_{1}+x_{2}, p_{21}=x_{1}, p_{22}=x_{2}-x_{1}$ and $p_{23}=x_{2}$. By Theorem 5.2 we see next that

$$
\begin{aligned}
\operatorname{dim}_{F} \mathcal{M} & =\sum_{j=1}^{3} l^{\mathcal{A}}\left(\mathcal{A} /\left\langle p_{11}, p_{2 j}\right\rangle\right)+2 \sum_{j=1}^{3} l^{\mathcal{A}}\left(\mathcal{A} /\left\langle p_{12}, p_{2 j}\right\rangle\right. \\
& =1+1+1+2+2+2=9 .
\end{aligned}
$$

5.4. Example. Some of the irreducible curves may be singular. Here is an example of such a linear 2-parameter system $\mathbf{f}=\left(f_{1}(\mathbf{x}), f_{2}(\mathbf{x})\right)$ in $\mathbb{A}^{5}$ :

$$
f_{1}\left(x_{1}, x_{2}\right)=\left(\begin{array}{ccccc}
x_{1} & x_{2} & 0 & 0 & 0 \\
0 & x_{1} & x_{2} & 0 & 0 \\
-1 & 0 & x_{1} & x_{2} & 0 \\
0 & 0 & 0 & x_{1} & x_{2} \\
0 & 0 & 0 & 1 & x_{1}
\end{array}\right), \quad f_{2}\left(x_{1}, x_{2}\right)=\left(\begin{array}{ccccc}
x_{1} & x_{2} & 0 & 0 & 0 \\
0 & x_{1} & x_{2} & 0 & 0 \\
0 & 0 & x_{1} & x_{2} & 0 \\
1 & 0 & 0 & x_{1} & x_{2} \\
0 & 0 & 0 & 0 & x_{1}
\end{array}\right)
$$

with the determinants $\operatorname{det} f_{1}=\left(x_{1}^{3}-x_{2}^{2}\right)\left(x_{1}^{2}-x_{2}\right)$ and $\operatorname{det} f_{2}=x_{1}\left(x_{1}^{4}-x_{2}^{3}\right)$. The irreducible components are then $p_{11}=x_{1}^{3}-x_{2}^{2}, p_{12}=x_{1}^{2}-x_{2}, p_{21}=x_{1}$ and $p_{22}=x_{1}^{4}-x_{2}^{3}$. The point $(0,0)$ is then singular point for the curves of $p_{11}$ and $p_{22}$. Next it follows that

$$
\begin{aligned}
i\left(p_{11}, p_{21}\right) & =l^{\mathcal{A}}\left(\mathcal{A} /\left\langle x_{1}^{3}-x_{2}^{2}, x_{1}\right\rangle\right)=l^{\mathcal{A}}\left(\mathcal{A} /\left\langle x_{1}, x_{2}^{2}\right\rangle\right)=2, \\
i\left(p_{11}, p_{22}\right) & =l^{\mathcal{A}}\left(\mathcal{A} /\left\langle x_{1}^{3}-x_{2}^{2}, x_{1}^{4}-x_{2}^{3}\right\rangle\right) \\
& =2 l^{\mathcal{A}}\left(\mathcal{A} /\left\langle x_{1}^{3}-x_{2}^{2}, x_{2}\right\rangle\right)+l^{\mathcal{A}}\left(\mathcal{A} /\left\langle x_{1}^{3}-x_{2}^{2}, x_{1}-x_{2}\right\rangle\right)=6+2=8,
\end{aligned}
$$


since $x_{1}^{4}-x_{2}^{3}=x_{1}\left(x_{1}^{3}-x_{2}^{2}\right)+x_{2}^{2}\left(x_{1}-x_{2}\right)$, and

$$
\begin{aligned}
i\left(p_{12}, p_{21}\right)= & l^{\mathcal{A}}\left(\mathcal{A} /\left\langle x_{1}^{2}-x_{2}, x_{1}\right\rangle\right)=l^{\mathcal{A}}\left(\mathcal{A} /\left\langle x_{1}, x_{2}\right\rangle\right)=1, \\
i\left(p_{12}, p_{22}\right)= & l^{\mathcal{A}}\left(\mathcal{A} /\left\langle x_{1}^{2}-x_{2}, x_{1}^{4}-x_{2}^{3}\right\rangle\right) \\
= & 2 l^{\mathcal{A}}\left(\mathcal{A} /\left\langle x_{1}^{2}-x_{2}, x_{2}\right\rangle\right)+l^{\mathcal{A}}\left(\mathcal{A} /\left\langle x_{1}^{2}-x_{2}, x_{1}-x_{2}\right\rangle\right) \\
& +l^{\mathcal{A}}\left(\mathcal{A} /\left\langle x_{1}^{2}-x_{2}, x_{1}+x_{2}\right\rangle\right) \\
= & 2+1+1=4,
\end{aligned}
$$

since $x_{1}^{4}-x_{2}^{3}=x_{1}^{2}\left(x_{1}^{2}-x_{2}\right)+x_{2}\left(x_{1}-x_{2}\right)\left(x_{1}+x_{2}\right)$. Because $l_{p_{i j}}\left(\mathcal{M}_{i}\right)=1$ for $i=1,2$ and $j=1,2$, we conclude that $\operatorname{dim}_{F}\left(\mathcal{M}_{1} \otimes_{\mathcal{A}} \mathcal{M}_{2}\right)=15$.

\section{Blow-up at a Singular Point}

The results in Theorem 4.3 and Theorem 5.2 provide a nice geometric interpretation of the dimension of the total root space at a point $\boldsymbol{\lambda}=\left(\lambda_{1}, \lambda_{2}, \ldots, \lambda_{n}\right)$, i.e. at the ideal $m=\left\langle x_{1}-\lambda_{1}, x_{2}-\lambda_{2}, \ldots, x_{n}-\lambda_{n}\right\rangle$, in the spectrum of a multiparameter system as long as all varieties associated with the irreducible factors of the $\operatorname{det} f_{i}$ are non-singular at that point. In the singular case the results still hold, but are not so intuitive anymore. As a remedy we propose to blow up the affine space $\mathbb{A}^{n}$ at the point $\boldsymbol{\lambda}$ (see [13], [18]).

Let us assume for simplicity that $\boldsymbol{\lambda}=(0,0, \ldots, 0) \in \mathbb{A}^{n}$; the blow up at another point is obtained by an affine change of coordinates. Consider the subvariety

$$
\mathbf{B}=\left\{(\mathbf{x}, \mathbf{y}) \mid x_{i} y_{j}=x_{j} y_{i}, 1 \leq i, j \leq n\right\} \subset \mathbb{A}^{n} \times \mathbb{P}^{n-1},
$$

where $\mathbb{P}^{n-1}$ is the $n$-1-dimensional projective space over $F$, and the projection map

$$
\pi: \mathbf{B} \rightarrow \mathbb{A}^{n} \times \mathbb{P}^{n-1} \stackrel{\text { proj }}{\longrightarrow} \mathbb{A}^{n}
$$

defined by $\pi(\mathbf{x}, \mathbf{y})=\mathbf{x}$. The 'diagonal' map

$$
\rho: \mathbb{A}^{n} /\{\boldsymbol{\lambda}\} \rightarrow \mathbf{B} / \pi^{-1}(\boldsymbol{\lambda}),
$$

given by $\rho(\mathbf{x})=(\mathbf{x}, \mathbf{x})$, is easily seen to be inverse to the corresponding restriction of $\pi$, and moreover

$$
\pi^{-1}(\boldsymbol{\lambda})=\{\boldsymbol{\lambda}\} \times \mathbb{P}^{n-1} \cong \mathbb{P}^{n-1} .
$$

For any line $L_{\mathbf{a}}$ passing through $\lambda$, described by the parametric equation $\mathbf{x}=\mathbf{a}$, one has $\rho(\mathbf{x})=(\mathbf{x}, \mathbf{a})$ for $t \neq 0$, and $\pi^{-1}\left(L_{\mathbf{a}}\right) \cap\left(\{\boldsymbol{\lambda}\} \times \mathbb{P}^{n-1}\right)=\{(\mathbf{0}, \mathbf{a})\}$. We may extend $\rho$ to

$$
\rho_{\mathbf{a}}: \mathbb{A}^{n} \rightarrow \mathbf{B}
$$

by defining $\rho(\boldsymbol{\lambda})=(\mathbf{0}, \mathbf{a})$. Choosing various lines, i.e. as a varies through $\mathbb{P}^{n-1}$, we get all possible points of $\boldsymbol{\lambda} \times \mathbb{P}^{n-1}$, thus blowing up $\boldsymbol{\lambda}$ to $\{\boldsymbol{\lambda}\} \times \mathbb{P}^{n-1}=\pi^{-1}(\boldsymbol{\lambda})$. This gives a bijective correspondence between lines through $\boldsymbol{\lambda}$ in $\mathbb{A}^{n}$ and points of the 'exceptional divisor' $E=\pi^{-1}(\boldsymbol{\lambda})$. Observe that $\mathbf{B}=\left(\mathbf{B} \backslash \pi^{-1}(\boldsymbol{\lambda})\right) \cup \pi^{-1}(\boldsymbol{\lambda})$ and that $\mathbf{B} \backslash \pi^{-1}(\boldsymbol{\lambda}) \cong \mathbb{A}^{n} \backslash\{\boldsymbol{\lambda}\}$ is irreducible. Hence the closure $\overline{\mathbf{B} \backslash \pi^{-1}(\lambda)}$ is irreducible as well. But $\pi^{-1}\left(L_{\mathbf{a}}\right)$, and thus also $\pi^{-1}\left(L_{\mathbf{a}}\right) \cap \pi^{-1}(\boldsymbol{\lambda})$, are in $\overline{\mathbf{B} \backslash \pi^{-1}(\boldsymbol{\lambda})}$ for every $\mathbf{a} \in \mathbb{P}^{n-1}$, so that $\pi^{-1}(\boldsymbol{\lambda}) \subset \overline{\mathbf{B} \backslash \pi^{-1}(\boldsymbol{\lambda})}$. Therefore, $\overline{\mathbf{B} \backslash \pi^{-1}(\boldsymbol{\lambda})}=\mathbf{B}$ and $\mathbf{B}$ is irreducible. 
If $Y \subset \mathbb{A}^{n}$ is a closed subvariety passing through $\lambda$, then the 'blow-up' of $Y$ at $\boldsymbol{\lambda}$ is the closure $Y_{\mathbf{B}}=\overline{\pi^{-1}(Y \backslash\{\boldsymbol{\lambda}\})}$ in $\mathbf{B}$. It is called the 'strict transform' of $Y$ under the blow-up $\pi: \mathbf{B} \rightarrow \mathbb{A}^{n}$ :

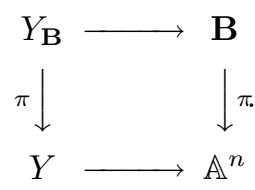

The total inverse image in $\mathbf{B}$ of the variety $Y$ of an irreducible polynomial $p(\mathbf{x}) \in$ $F[\mathbf{x}]$ passing through $\mathbf{0}$ in $\mathbb{A}^{n}$ is

$$
\pi^{-1}(Y)=\left\{(\mathbf{x}, \mathbf{y}) \mid p(\mathbf{x})=0 ; x_{i} y_{j}=x_{j} y_{i}, 1 \leq i, j \leq n\right\} \subset \mathbb{A}^{n} \times \mathbb{P}^{n-1} .
$$

The projective space $\mathbb{P}^{n-1}$ is covered by the $n$ open sets $U_{i}=\left\{\mathbf{y} \mid y_{i} \neq 0\right\}$. If $y_{1} \neq 0$, then we may set $y_{1}=1$ and use the remaining coordinates as affine parameters, so that

$$
\pi^{-1}(Y) \cap\left(\mathbb{A}^{n} \times U_{1}\right)=\left\{(\mathbf{x}, \mathbf{z}) \mid p(\mathbf{x})=0, \mathbf{x}=x_{1}(1, \mathbf{z})\right\} \subset \mathbb{A}^{2 n-1} .
$$

The substitution $\mathbf{x}=x_{1}(1, \mathbf{z})$ then gives

$$
p(\mathbf{x})=x_{1}^{e_{p}} q\left(x_{1}, \mathbf{z}\right),
$$

where $q$ is irreducible and not divisible by $x_{1}$, and $e_{p}$ is the largest power of the maximal ideal $m$ of $\mathcal{A}$ containing $p(\mathbf{x})$. The two irreducible components give $E=$ $\{(\mathbf{x}, \mathbf{z}) \mid \mathbf{x}=\mathbf{0}\}$ and $B_{Y}=\left\{(\mathbf{x}, \mathbf{z}) \mid q\left(x_{1}, \mathbf{z}\right)=0, \mathbf{x}=x_{1}(1, \mathbf{z})\right\}$, so that $B_{Y} \cap E=$ $\{\mathbf{z} \mid q(0, \mathbf{z})=0\}$ is a variety of dimension $n-2$.

6.1. Proposition. Let $p_{1}(\mathbf{x}), p_{2}(\mathbf{x}), \ldots, p_{n}(\mathbf{x})$ be a regular sequence in $F[\mathbf{x}]$ and let $Y_{1}, Y_{2}, \ldots, Y_{n}$ be the corresponding hypersurfaces in $\mathbb{A}^{n}$. Then $E \bigcap_{i=1}^{n} Y_{\mathbf{B}}$ is a finite set and

$$
i\left(\boldsymbol{\lambda} ; Y_{1} Y_{2} \ldots Y_{n} ; \mathbb{A}^{n}\right)=\prod_{i=1}^{n} e_{\boldsymbol{\lambda}}\left(Y_{i}\right)+\sum_{\boldsymbol{\mu} \in E} i\left(\boldsymbol{\mu} ; \mathbf{B}_{Y_{1}}, \mathbf{B}_{Y_{2}}, \ldots, \mathbf{B}_{Y_{n}} ; \mathbf{B}\right)
$$

where $e_{\boldsymbol{\lambda}}\left(Y_{i}\right)=e_{p_{i}}$ is the largest power of the maximal ideal $m$ of $\mathcal{A}$ containing $p_{i}(\mathbf{x})$.

Proof. If $p_{1}(\mathbf{x}), p_{2}(\mathbf{x}), \ldots, p_{n}(\mathbf{x})$ form a regular sequence of polynomials in $F[\mathbf{x}]$, then the intersection multiplicity

$$
i\left(\boldsymbol{\lambda} ; Y_{1} Y_{2} \ldots Y_{n} ; \mathbb{A}^{n}\right)=l^{\mathcal{A}}\left(\mathcal{A} /\left\langle p_{1}, p_{2}, \ldots, p_{n}\right\rangle\right)
$$

of the corresponding hypersurfaces $Y_{1}, Y_{2}, \ldots, Y_{n}$ at the point $\boldsymbol{\lambda}$ in $\mathbb{A}^{n}$ is finite, and hence $E \bigcap_{i=1}^{n} \mathbf{B}_{Y_{i}}$ is a finite set. Moreover, if $\pi: \mathbf{B} \rightarrow \mathbb{A}^{n}$ is the blow-up of $\mathbb{A}^{n}$ at the point $\boldsymbol{\lambda}$ with the exceptional divisor $E$, then the inverse image divisor of $Y_{i}$ on $B$ is $\pi^{*}\left(Y_{i}\right)=e_{\boldsymbol{\lambda}}\left(Y_{i}\right) E+\mathbf{B}_{Y_{i}}$ [8, p. 82]. The assertion now follows from [8, p. 124]. See also [18, IV.3].

6.2. Example. As an illustration let us consider again the same 2-parameter system $\mathbf{f}$ in $\mathbb{A}^{5}$ as in Example 5.4:

$$
f_{1}\left(x_{1}, x_{2}\right)=\left(\begin{array}{ccccc}
x_{1} & x_{2} & 0 & 0 & 0 \\
0 & x_{1} & x_{2} & 0 & 0 \\
-1 & 0 & x_{1} & x_{2} & 0 \\
0 & 0 & 0 & x_{1} & x_{2} \\
0 & 0 & 0 & 1 & x_{1}
\end{array}\right), \quad f_{2}\left(x_{1}, x_{2}\right)=\left(\begin{array}{ccccc}
x_{1} & x_{2} & 0 & 0 & 0 \\
0 & x_{1} & x_{2} & 0 & 0 \\
0 & 0 & x_{1} & x_{2} & 0 \\
1 & 0 & 0 & x_{1} & x_{2} \\
0 & 0 & 0 & 0 & x_{1}
\end{array}\right),
$$


with the determinants $\operatorname{det} f_{1}=\left(x_{1}^{3}-x_{2}^{2}\right)\left(x_{1}^{2}-x_{2}\right)$ and $\operatorname{det} f_{2}=x_{1}\left(x_{1}^{4}-x_{2}^{3}\right)$. Then, with $x_{1} z=x_{2}$, one obtains $p_{11}=x_{1}^{2}\left(x_{1}-z^{2}\right), p_{12}=x_{1}\left(x_{1}-z\right), p_{21}=x_{1}$ and $p_{22}=x_{1}^{3}\left(x_{1}-z^{3}\right)$, and we see that

$$
\begin{aligned}
& i\left(Y_{11}, Y_{21}\right)=e\left(Y_{11}\right) e\left(Y_{21}\right)+i\left(Z_{11}, Z_{21}\right)=2+0=2, \\
& i\left(Y_{11}, Y_{22}\right)=e\left(Y_{11}\right) e\left(Y_{22}\right)+i\left(Z_{11}, Z_{22}\right)=6+2=8, \\
& i\left(Y_{12}, Y_{21}\right)=e\left(Y_{12}\right) e\left(Y_{21}\right)+i\left(Z_{12}, Z_{21}\right)=1+0=1, \\
& i\left(Y_{12}, Y_{22}\right)=e\left(Y_{12}\right) e\left(Y_{22}\right)+i\left(Z_{12}, Z_{22}\right)=3+1=4 .
\end{aligned}
$$

Since all $l_{p_{i j}}\left(M_{i}\right)=1$ we conclude that $\operatorname{dim}_{F} M_{1} \otimes_{A} M_{2}=15$, which coincides with the result obtained in Example 5.4.

\section{7. (In)decomposability of the Associated Modules}

7.1. Proposition. If the module $\mathcal{M}=\mathcal{M}_{1} \otimes_{\mathcal{A}} \mathcal{M}_{2} \otimes_{\mathcal{A}} \cdots \otimes_{\mathcal{A}} \mathcal{M}_{n}$ is indecomposable then all the modules $\mathcal{M}_{i}$ are indecomposable.

Proof. It is clear that if $\mathcal{M}_{i}$ is decomposable then $\mathcal{M}$ is decomposable, since $\oplus$ and $\otimes_{\mathcal{A}}$ are distributive.

7.2. Proposition. If $m \in \sigma(\mathbf{f})$ is simple then $\mathcal{M}$, and also each $\mathcal{M}_{i}$, is indecomposable.

Proof. The decomposition of $\mathcal{M}=\mathcal{K} \oplus \mathcal{L}$ induces a decomposition of $\mathcal{M}^{(0)}=$ $\mathcal{K}^{(0)} \oplus \mathcal{L}^{(0)}$, where $\mathcal{M}^{(0)}=\mathcal{M} / m \mathcal{M}$. Since $\mathcal{M}^{(0)}$ is one-dimensional, one of $\mathcal{K}^{(0)}$ and $\mathcal{L}^{(0)}$ is 0 . By the Nakayama Lemma, then, one of the modules $\mathcal{K}$ or $\mathcal{L}$ is 0 . The proof for $\mathcal{M}_{i}$ is the same.

In general however, the converse of Proposition 7.1 does not hold:

7.3. Example. Consider the two-parameter system

$$
f_{1}(\mathbf{x})=\left(\begin{array}{cccc}
x_{1} & 0 & x_{1} & x_{2} \\
0 & x_{1} & 0 & 0 \\
1 & 0 & x_{1} & 0 \\
0 & 1 & 0 & x_{1}
\end{array}\right) \text { and } f_{2}(\mathbf{x})=\left(\begin{array}{cccc}
x_{2} & 0 & x_{2} & x_{1} \\
0 & x_{2} & 0 & 0 \\
1 & 0 & x_{2} & 0 \\
0 & 1 & 0 & x_{2}
\end{array}\right)
$$

Then $\operatorname{det} f_{1}(\mathbf{x})=x_{1}^{3}\left(x_{1}-1\right)$ and $\operatorname{det} f_{2}(\mathbf{x})=x_{2}^{3}\left(x_{2}-1\right)$. Because $\Delta_{0}$ is invertible it follows by Theorem 5.1 that the system $\mathbf{f}=\left(f_{1}, f_{2}\right)$ is regular. All the irreducible factors of the determinants $\operatorname{det} f_{1}$ and $\operatorname{det} f_{2}$ are linear. Counting the multiplicities of these irreducible factors, we see from Theorem 5.2 that the root subspace at $(0,0)$ has dimension 9.

One checks directly that

$$
\mathcal{M}_{1}^{0(0)}=\left\{e_{00} \otimes\left(\begin{array}{l}
0 \\
0 \\
\alpha \\
\beta
\end{array}\right): \alpha, \beta \in F\right\}
$$

and

$$
\mathcal{M}_{1}^{0(1)}=\left\{e_{00} \otimes\left(\begin{array}{l}
\alpha \\
\beta \\
\gamma \\
\delta
\end{array}\right)+e_{01} \otimes\left(\begin{array}{l}
0 \\
0 \\
\varepsilon \\
\alpha
\end{array}\right)-e_{10} \otimes\left(\begin{array}{l}
0 \\
0 \\
\alpha \\
\beta
\end{array}\right): \alpha, \beta, \gamma, \delta, \varepsilon \in F\right\}
$$


Let $\mathcal{U}$ be the subcomodule of $\mathcal{M}_{1}^{0}$ 'generated' by the element

$$
u=e_{00} \otimes\left(\begin{array}{l}
1 \\
0 \\
0 \\
0
\end{array}\right)+e_{01} \otimes\left(\begin{array}{l}
0 \\
0 \\
0 \\
1
\end{array}\right)-e_{10} \otimes\left(\begin{array}{l}
0 \\
0 \\
1 \\
0
\end{array}\right)
$$

Then

$$
\mathcal{U}=\left\{e_{00} \otimes\left(\begin{array}{l}
\alpha \\
0 \\
\beta \\
\gamma
\end{array}\right)+e_{01} \otimes\left(\begin{array}{l}
0 \\
0 \\
0 \\
\alpha
\end{array}\right)-e_{10} \otimes\left(\begin{array}{l}
0 \\
0 \\
\alpha \\
0
\end{array}\right): \alpha, \beta, \gamma \in F\right\}
$$

Since $\mathcal{U}$ is the smallest subcomodule of $\mathcal{M}_{1}^{0}$ containing $u$, it is indecomposable. Note also that $\mathcal{M}_{1}^{0(0)} \subset \mathcal{U}$ and therefore $\mathcal{U}^{(0)}=\mathcal{M}_{1}^{0(0)}$. Suppose that $\mathcal{M}_{1}^{0}$ is decomposable, i.e. $\mathcal{M}_{1}^{0}=\mathcal{K} \oplus \mathcal{L}$. Then $\mathcal{U}=(\mathcal{U} \cap \mathcal{K}) \oplus(\mathcal{U} \cap \mathcal{L})$. From the relation $\mathcal{U}^{(0)}=\mathcal{M}_{1}^{0(0)}$, it follows that $\mathcal{U}^{(0)}=\mathcal{K}^{(0)} \oplus \mathcal{L}^{(0)}$, and hence either $\mathcal{K}^{(0)}=0$ or $\mathcal{L}^{(0)}=0$. But then it follows by the dual Nakayama Lemma [12] that either $\mathcal{K}=0$ or $\mathcal{L}=0$. Hence $\mathcal{M}_{1}^{0}$ is indecomposable. The proof that $\mathcal{M}_{2}^{0}$ is indecomposable is the same, only the indices $i=1$ and $i=2$ are interchanged.

Let $R$ be the root subspace of the associated system $\Gamma$ at $\boldsymbol{\lambda}=(0,0)$. Obviously $R$ is invariant for both $\Gamma_{1}$ and $\Gamma_{2}$. We use the main results of [15] to construct a basis $\mathcal{B}$ for $R$ such that the pair $\left(\Gamma_{1}, \Gamma_{2}\right)$ restricted to $R$ is in the canonical form

$$
\left.\Gamma_{1}\right|_{R}=\left(\begin{array}{ccccccccc}
0 & 0 & 0 & 1 & 0 & 0 & 0 & 0 & 0 \\
0 & 0 & 0 & 0 & 1 & 0 & 0 & 0 & 0 \\
0 & 0 & 0 & 0 & 0 & 1 & 0 & 0 & 0 \\
0 & 0 & 0 & 0 & 0 & 0 & 0 & 0 & 0 \\
0 & 0 & 0 & 0 & 0 & 0 & 0 & 0 & 0 \\
0 & 0 & 0 & 0 & 0 & 0 & 0 & 1 & 0 \\
0 & 0 & 0 & 0 & 0 & 0 & 0 & 0 & 0 \\
0 & 0 & 0 & 0 & 0 & 0 & 0 & 0 & 0 \\
0 & 0 & 0 & 0 & 0 & 0 & 0 & 0 & 0
\end{array}\right)
$$

and

$$
\left.\Gamma_{2}\right|_{R}=\left(\begin{array}{ccccccccc}
0 & 0 & 0 & 0 & 1 & 0 & 0 & 0 & 0 \\
0 & 0 & 0 & 0 & 0 & 1 & 0 & 0 & 0 \\
0 & 0 & 0 & 0 & 0 & 0 & 1 & 0 & 0 \\
0 & 0 & 0 & 0 & 0 & 0 & 0 & 0 & 0 \\
0 & 0 & 0 & 0 & 0 & 0 & 0 & 1 & 0 \\
0 & 0 & 0 & 0 & 0 & 0 & 0 & 0 & 0 \\
0 & 0 & 0 & 0 & 0 & 0 & 0 & 0 & 0 \\
0 & 0 & 0 & 0 & 0 & 0 & 0 & 0 & 0 \\
0 & 0 & 0 & 0 & 0 & 0 & 0 & 0 & 0
\end{array}\right) .
$$

Now it is easy to check that $R=R_{1} \oplus R_{2}$, where $R_{1}$ is spanned by the first 8 vectors of the basis $\mathcal{B}$ and $R_{2}$ is spanned by the last vector in $\mathcal{B}$, and that both $R_{1}$ and $R_{2}$ are invariant for $\Gamma_{1}$ and $\Gamma_{2}$. Then $R$ is decomposable as a module over the algebra generated by $\Gamma_{1}$ and $\Gamma_{2}$. Since $R=\varepsilon^{\dagger}\left(\mathcal{M}_{1}^{(0)} \otimes^{\mathcal{B}_{0}} \mathcal{M}_{2}^{(0)}\right)$, by the main theorem of [10] it follows that $\mathcal{M}_{1}^{(0)} \otimes^{\mathcal{B}_{0}} \mathcal{M}_{2}^{(0)}$, and thus also $\mathcal{M}_{1} \otimes_{\mathcal{A}} \mathcal{M}_{2}$, is decomposable. 


\section{REFERENCES}

1. M.F. Atiyah and I.G. MacDonald, Introduction to Commutative Algebra, Addison-Wesley Publ. Co., 1969. MR 39:4129

2. F.V. Atkinson, Multiparameter Eigenvalue Problems, Academic Press, 1972. MR 56:9291

3. P.A. Binding and P.J. Browne, Two Parameter Eigenvalue Problems for Matrices, Lin. Alg. Appl., 113:139-157, 1989. MR 89m:47012

4. W. Bruns and J. Herzog, Cohen-Macaulay Rings, Cambridge Univ. Press, $1993 . \quad$ MR 95h: 13020

5. A.J. Cook and A.D. Thomas, Line Bundles and Homogeneous Matrices, Quart. J. Math. Oxford, (2)30:423-429, 1979. MR 81e:14031

6. A.C. Dixon, Note on the Reduction of Ternary Quartic to a Symmetrical Determinant, Proc. Cambridge Phil. Soc., 2:350-351, 1900-1902.

7. M. Faierman, Two-parameter Eigenvalue Problems in Ordinary Differential Equations, volume 205 of Pitman Research Notes in Mathematics, Longman Scientific and Technical, Harlow, U.K., 1991. MR 93b:47095

8. W. Fulton, Intersection Theory, Springer-Verlag, 1984. MR 85k:14004

9. G.A. Gadzhiev, Introduction to Multiparameter Spectral Theory (in Russian), Azerbaijan State University, Baku, 1987.

10. L. Grunenfelder and T. Košir, An Algebraic Approach to Multiparameter Eigenvalue Problems, Trans. Amer. Math. Soc. 348: 2983-2998, 1996. MR 96j:47015

11. L. Grunenfelder and T. Košir, Coalgebras and Spectral Theory in One and Several Parameters, Recent Developments in Operator Theory and Its Applications (Manitoba, 1994), Operator Theory: Advances and Applications, vol. 87, Birkhäuser Verlag, 1996, pp. 177-192. CMP 96:15

12. L. Grunenfelder and T. Košir, Koszul Cohomology for Finite Families of Comodule Maps and Applications, Comm. in Alg 25: 459-479, 1997. CMP 97:06

13. R. Hartshorne, Algebraic Geometry, Springer-Verlag, 1977. MR 57:3116

14. H.(G.A.) Isaev, Lectures on Multiparameter Spectral Theory, Dept. of Math. and Stats., University of Calgary, 1985.

15. T. Košir, Kronecker Bases for Linear Matrix Equations, with Application to Two-Paramater Eigenvalue Problems, Lin. Alg. Appl. 249: 259-288, 1996. MR 97g:15016

16. H. Matsumura, Commutative Algebra, Benjamin/Cummings Publ., Reading, Mass., 2nd edition, 1980. MR 82i: 13003

17. J.P. Serre, Algèbre Locale - Multiplicités, volume 11 of Lect. Notes in Math., Springer-Verlag, 1965. MR 34:1352

18. I.R. Shafarevich, Basic Algebraic Geometry, Springer-Verlag, 1974. MR 51:3163

19. B.D. Sleeman, Multiparameter Spectral Theory in Hilbert Space, volume 22 of Pitman Research Notes in Mathematics, Pitman Publ. Ltd., London U.K., Belmont U.S.A., 1978. MR 81h:47004

20. V. Vinnikov, Complete Description of Determinantal Representations of Smooth Irreducible Curves, Lin. Alg. Appl., 125:103-140, 1989. MR 90m:14028

21. H. Volkmer, Multiparameter Eigenvalue Problems and Expansion Theorems, volume 1356 of Lecture Notes in Mathematics, Springer-Verlag, Berlin, New York, 1988. MR 90d:47021

Department of Mathematics, Statistics and Computing Science, Dalhousie UniverSity, Halifax, Nova Scotia, Canada, B3H 3J5

E-mail address: luzius@cs.dal.ca

Department of Mathematics, University of Ljubljana, Jadranska 19, 1000 Ljubljana, SLOVENIA

E-mail address: tomaz.kosir@fmf.uni-lj.si 